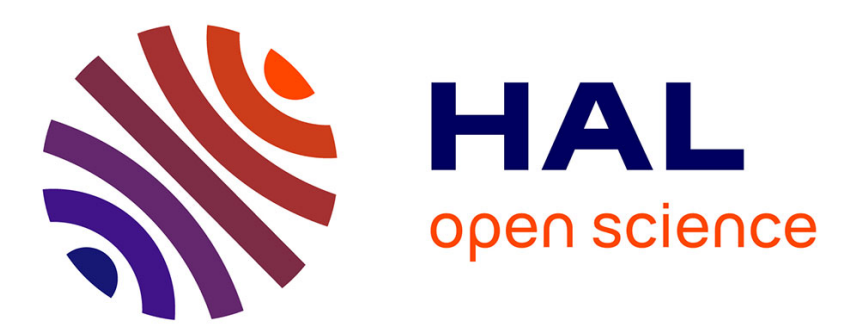

\title{
Hierarchical propagation of probabilistic and non-probabilistic uncertainty in the parameters of a risk model
}

Nicola Pedroni, Enrico Zio, Elisa Ferrario, Alberto Pasanisi, Mathieu Couplet

\section{- To cite this version:}

Nicola Pedroni, Enrico Zio, Elisa Ferrario, Alberto Pasanisi, Mathieu Couplet. Hierarchical propagation of probabilistic and non-probabilistic uncertainty in the parameters of a risk model. Computers \& Structures, 2013, Online, pp.1-15. 10.1016/j.compstruc.2013.02.003 . hal-00839639

\section{HAL Id: hal-00839639 \\ https://hal-centralesupelec.archives-ouvertes.fr/hal-00839639}

Submitted on 28 Jun 2013

HAL is a multi-disciplinary open access archive for the deposit and dissemination of scientific research documents, whether they are published or not. The documents may come from teaching and research institutions in France or abroad, or from public or private research centers.
L'archive ouverte pluridisciplinaire HAL, est destinée au dépôt et à la diffusion de documents scientifiques de niveau recherche, publiés ou non, émanant des établissements d'enseignement et de recherche français ou étrangers, des laboratoires publics ou privés. 


\title{
Hierarchical propagation of probabilistic and non-probabilistic
}

\section{uncertainty in the parameters of a risk model}

\author{
N. Pedroni ${ }^{1}$, E. Zio ${ }^{1,2, *}$, E. Ferrario ${ }^{2}$, A. Pasanisi ${ }^{3}$, M. Couplet $^{3}$ \\ *Corresponding author \\ * Chair of system science and the energetic challenge, Electricitè de France-Ecole Centrale Paris and Supelec \\ ${ }^{1}$ Energy Department, Politecnico di Milano \\ Via Ponzio, 34/3 - 20133 Milano, Italy \\ Fax: +39-02-2399-6309; Phone: +39-02-2399-6340 \\ E-mail address: nicola.pedroni@mail.polimi.it \\ E-mail address: enrico.zio@polimi.it \\ ${ }^{2}$ Ecole Centrale Paris \\ Grande Voie des Vignes, 92295, Chatenay Malabry-Cedex, France \\ Fax: +33-01-41-13-12-72; Phone: +33-01-41-13-16-06 \\ E-mail address: enrico.zio@ecp.fr \\ ${ }^{3}$ Electricité de France, Chatou, France \\ E-mail address: alberto.pasanisi@edf.fr
}

\begin{abstract}
We consider a model for the risk-based design of a flood protection dike, and use probability distributions to represent aleatory uncertainty and possibility distributions to describe the epistemic uncertainty associated to the poorly known parameters of such probability distributions.

A hybrid method is introduced to hierarchically propagate the two types of uncertainty, and the results are compared with those of a Monte Carlo-based Dempster-Shafer approach employing independent random sets and a purely probabilistic, two-level Monte Carlo approach: the risk estimates produced are similar to those of the Dempster-Shafer method and more conservative than those of the two-level Monte Carlo approach.
\end{abstract}

Keywords: hierarchical uncertainty, possibility distributions, fuzzy interval analysis, two-level Monte Carlo method, dependences, flood protection dike. 


\section{Introduction}

In risk analysis, uncertainty is typically distinguished into two types: randomness due to inherent variability in the system behavior and imprecision due to lack of knowledge and information on the system. The former type of uncertainty is often referred to as objective, aleatory, stochastic whereas the latter is often referred to as subjective, epistemic, state of knowledge [1], [2].

We are interested in the framework of two hierarchical levels of uncertainty, referred to as "twolevel" setting [3]: the models of the aleatory events (e.g., the failure of a mechanical component or the variation of its geometrical dimensions and material properties) contain parameters (e.g., probabilities, failure rates,...) that are epistemically uncertain because known with poor precision by the analyst.

Both the aleatory and epistemic uncertainties in the two-level framework can be represented by probability distributions, and propagated by two-level (or double loop) Monte Carlo (MC) simulation [4]: in the outer simulation loop, the values of the parameters affected by epistemic uncertainty are sampled and fed onto the probability distributions of the inner loop where the aleatory variables are sampled [5], [6].

In some cases, the imprecise knowledge, incomplete information and scarce data impair the probabilistic representation of epistemic uncertainty. A number of alternative representation frameworks have been proposed to handle such cases [7], e.g., fuzzy set theory [8], DempsterShafer theory of evidence [9]-[14], possibility theory [15]-[18] and interval analysis [19]-[21].

In this paper, we use probability distributions to describe the first level aleatory uncertainty and possibility distributions to describe the second level epistemic uncertainty in the parameters of such probability distributions [15]-[18].

For the propagation of the hybrid (probabilistic and possibilistic) uncertainty representation, the MC technique [22], [23] is combined with the extension principle of fuzzy set theory [24]-[33], within a 
"two-level" hierarchical setting [16], [34]-[39]. This is done by i) fuzzy interval analysis to process the uncertainty described by possibility distributions, ii) repeated MC sampling of the random variables to process aleatory uncertainty [16], [24], [29].

The joint hierarchical propagation of probabilistic and possibilistic representations of uncertainty is applied to a model for the risk-based design of a flood protection dike developed as a realistic benchmark for uncertainty modeling [3]; the effectiveness of the propagation method is compared to that of: i) a Monte Carlo (MC)-based Dempster-Shafer (DS) approach employing Independent Random Sets (IRSs) (i.e., where the epistemically uncertain parameters are represented by discrete focal sets that are randomly and independently sampled by MC) ${ }^{1}$ [40]-[50], ii) a traditional twolevel MC approach [2], [4], [6]. To the best of the authors' knowledge, this is the first time that the above mentioned methods are systematically compared with reference to risk assessment problems where hybrid uncertainty is separated into two hierarchical levels.

The remainder of the paper is organized as follows. In Section 2, the hybrid method for uncertainty propagation is described; in Section 3, the flood model is presented; in Section 4, the results of the joint hierarchical propagation of aleatory and epistemic uncertainties through the model of Section 3, and the comparison with the MC-based DS-IRS and two-level MC approaches are reported and commented; in Section 5, conclusions are provided. The details about the hybrid, MC-based DSIRS and two-level MC computational procedures are given in Appendices A, B and C, respectively.

\section{Joint hierarchical propagation of aleatory and epistemic}

\section{uncertainties in a "two-level" framework}

In all generality, we consider a model whose output is a function $Z=f\left(Y_{1}, Y_{2}, \ldots, Y_{n}\right)$ of $n$ uncertain variables $Y_{i}, i=1, \cdots, n$, ordered in such a way that the first $k, Y_{1}, Y_{2}, \ldots, Y_{j}, \ldots, Y_{k}$, are

\footnotetext{
${ }^{1}$ In the following, this method will be referred to as "MC-based DS-IRS approach" for brevity.
} 
"probabilistic", i.e., their uncertainty is described by probability distributions $p_{Y_{1}}\left(y_{1} \mid \boldsymbol{\theta}_{1}\right), p_{Y_{2}}\left(y_{2} \mid \boldsymbol{\theta}_{2}\right), \ldots, p_{Y_{j}}\left(y_{j} \mid \boldsymbol{\theta}_{j}\right), \ldots, p_{Y_{k}}\left(y_{k} \mid \boldsymbol{\theta}_{k}\right)$, where $\boldsymbol{\theta}_{j}=\left\{\boldsymbol{\theta}_{j, 1}, \boldsymbol{\theta}_{j, 2}, \ldots, \boldsymbol{\theta}_{j, m_{j}}\right\}, j=1,2, \cdots, k$, are the vectors of the corresponding internal parameters, and the last $n-k, Y_{k+1}, Y_{k+2}, \ldots, Y_{l}, \ldots, Y_{n}$, are "purely possibilistic", i.e., their uncertainty is epistemic and represented by the possibility distributions $\pi^{Y_{k+1}}\left(y_{k+1}\right), \pi^{Y_{k+2}}\left(y_{k+2}\right), \ldots, \pi^{Y_{l}}\left(y_{l}\right), \ldots, \pi^{Y_{n}}\left(y_{n}\right)$.

In a "two-level" framework, the parameters $\boldsymbol{\theta}_{j}, j=1,2, \cdots, k$, are themselves affected by epistemic uncertainty. We describe these uncertainties by possibility distributions $\boldsymbol{\pi}^{\boldsymbol{\theta}_{j}}\left(\boldsymbol{\theta}_{j}\right)=\left\{\boldsymbol{\pi}^{\theta_{j, 1}}\left(\theta_{j, 1}\right), \pi^{\theta_{j, 2}}\left(\theta_{j, 2}\right), \ldots, \pi^{\theta_{j, m_{j}}}\left(\theta_{j, m_{j}}\right)\right\}, j=1,2, \cdots, k$. For clarification by way of example, we may consider $Y \sim N(\mu, \sigma)=N(\boldsymbol{\theta})=N\left(\theta_{1}, \theta_{2}\right)$, where the parameter $\mu=\theta_{1}$ has a triangular possibility distribution with core $\{c\}$ and support $[a, b]$, and parameter $\sigma=\theta_{2}$ has a triangular possibility distribution with core $\{f\}$ and support $[e, d]$.

The propagation of the hybrid uncertainty can be performed by combining the Monte Carlo (MC) technique [22], [23] with the extension principle of fuzzy set theory [24]-[33] by means of the following two main steps [16], [34]-[39]:

i. fuzzy interval analysis to process epistemic uncertainty;

ii. repeated MC sampling of the random variables to process aleatory uncertainty.

Technical details about the operative steps of the procedure are given in Appendix A.

The method produces $m$ possibility distributions $\pi_{i}^{f}(z), i=1,2, \ldots, m$, for the output variable $Z=f\left(Y_{1}, Y_{2}, \ldots, Y_{n}\right)$ (where $m$ is the number of random samples of the aleatory variables drawn by MC). Then, for each set $A$ contained in the universe of discourse $U_{Z}$ of $Z$, it is possible to obtain the possibility measure $\Pi_{i}^{f}(A)$ and the necessity measure $N_{i}^{f}(A)$ from $\pi_{i}^{f}(z), i=1,2, \ldots, m$, by: $\Pi_{i}^{f}(A)=\max _{z \in A}\left\{\pi_{i}^{f}(z)\right\}$ 
$N_{i}^{f}(A)=\inf _{z \notin A}\left\{1-\pi_{i}^{f}(z)\right\}=1-\Pi_{i}^{f}(\bar{A}) \quad \forall A \subseteq U_{Z}$

The $m$ different realizations of possibility and necessity can then be combined to obtain the belief $\operatorname{Bel}(A)$ and the plausibility $\operatorname{Pl}(A)$ for any set $A$, respectively [15]:

$\operatorname{Bel}(A)=\sum_{i=1}^{m} p_{i} N_{i}^{f}(A)$

$\operatorname{Pl}(A)=\sum_{i=1}^{m} p_{i} \Pi_{i}^{f}(A)$

where $p_{i}$ is the probability of sampling the $i-t h$ realization of the random variable vector $\left(Y_{1}, Y_{2} \cdots, Y_{k}\right)$ : if $m$ realizations are generated by plain random sampling, then $p_{i}$ is simply $1 / m$. For each set $A$, this technique thus computes the probability-weighted average of the possibility measures associated with each output fuzzy interval.

The likelihood of the value $f(Y)$ passing a given threshold $z$ can then be computed by considering the belief and the plausibility of the set $A=(-\infty, z]$; in this respect, $\operatorname{Bel}(f(Y) \in(-\infty, z])$ and $P l(f(Y) \in(-\infty, z])$ can be interpreted as bounding, average cumulative distributions $\underline{F}(z)=\operatorname{Bel}(f(Y) \in(-\infty, z]), \bar{F}(z)=P l(f(Y) \in(-\infty, z])[15]$.

Let the core and the support of a possibilistic distribution $\pi^{f}(z)$ be the crisp sets of all points of $U_{Z}$ such that $\pi^{f}(z)$ is equal to 1 and nonzero, respectively. Considering a generic value $z$ of $f(Y)$, it is $P l(f(Y) \in(-\infty, z])=1$ if and only if $\Pi_{i}^{f}(f(Y) \in(-\infty, z])=1, \forall i=1, \cdots, m$, that is, for $z>z^{*}=\max _{i}\left\{\inf \left(\operatorname{core}\left(\pi_{i}^{f}\right)\right)\right\} . \quad$ Similarly, $\quad P l(f(Y) \in(-\infty, z])=0 \quad$ if $\quad$ and $\quad$ only $\quad$ if $\Pi_{i}^{f}(f(Y) \in(-\infty, z])=0 \quad \forall i=1, \cdots, m$, that is, for $z \leq z^{*}=\min _{i}\left\{\inf \left(\operatorname{support}\left(\pi_{i}^{f}\right)\right)\right\}$.

Finally, one way to estimate the total uncertainty on $f(Y)$ is to provide a confidence interval at a given level of confidence, taking the lower and upper bounds from $\operatorname{Pl}(f(Y) \in(-\infty, z])$ and $\operatorname{Bel}(f(Y) \in(-\infty, z]), \quad$ respectively [15]. On the other hand, $\operatorname{Bel}(f(Y) \in(-\infty, z])$ and 
$P l(f(Y) \in(-\infty, z])$ cannot convey any information on the prediction that $f(Y)$ lies within a given interval $\left[z_{1}, z_{2}\right]$, since neither $\operatorname{Bel}\left(f(Y) \in\left[z_{1}, z_{2}\right]\right)$ nor $P l\left(f(Y) \in\left[z_{1}, z_{2}\right]\right)$ can be expressed in terms of $\operatorname{Bel}(f(Y) \in(-\infty, z])$ and $\operatorname{Pl}(f(Y) \in(-\infty, z])$, respectively.

\section{Case study: flood protection risk-based design}

The case study deals with the design of a protection dike in a residential area closely located to a river with potential risk of floods. Two issues of concern are: i) high construction and annual maintenance costs of the dike; ii) uncertainty in the natural phenomenon of flooding. Then, the different design options must be evaluated within a flooding risk analysis framework accounting for uncertainty.

In Section 3.1, a short description of the model for flood protection dike design is given; in Section 3.2, the uncertain variables of the model are described.

\subsection{The model}

The maximal water level of the river (i.e., the output variable of the model, $Z_{c}$ ) is given as a function of several (and some uncertain) parameters (i.e., the input variables of the model) [3]:

$$
Z_{c}=Z_{v}+\left(\frac{Q}{K_{s} * B * \sqrt{\left(Z_{m}-Z_{v}\right) / L}}\right)^{3 / 5}
$$

where:

- $Q$ is the yearly maximal water discharge $\left(\mathrm{m}^{3} / \mathrm{s}\right)$;

- $Z_{m}$ and $Z_{v}$ are the riverbed levels ( $\mathrm{m}$ asl) at the upstream and downstream part of the river under investigation, respectively;

$-K_{s}$ is the Strickler friction coefficient; 
- $\quad B$ and $L$ are the width and length of the river part (m), respectively.

The input variables are classified as follows:

- Constants: $B=300 \mathrm{~m}, L=5000 \mathrm{~m}$.

- Uncertain variables: $Q, Z_{m}, Z_{v}, K_{s}$.

\subsection{The input variables: physical description and representation of the associated uncertainty}

The input variables are affected by aleatory and epistemic uncertainties. The aleatory part of the uncertainty is described by probability distributions of defined shape (e.g., normal, exponential, ...). The parameters of the probability distributions describing the aleatory uncertainty are themselves affected by epistemic uncertainty represented in terms of possibility distributions.

In this Section, a detailed description of the uncertain input variables is given together with the explanation of the reasons underlying the choices of their description by probability and possibility distributions. In particular, in Section 3.2.1, the yearly maximal water flow $Q$ is discussed; in Section 3.2.2, the upstream and downstream riverbed levels $Z_{m}$ and $Z_{v}$ are presented; finally, in Section 3.2.3, the Strickler friction coefficient $K_{s}$ is described.

\subsubsection{The yearly maximal water flow, $Q$}

The Gumbel distribution $\operatorname{Gum}(q \mid \alpha, \beta)$ is a well-established probabilistic (aleatory) model for maximal flows [3]:

$\operatorname{Gum}(q \mid \alpha, \beta)=\frac{1}{\beta} \exp \left[-\exp \left(\frac{q-\alpha}{\beta}\right)\right] \exp \left[\frac{\alpha-q}{\beta}\right]$

The extreme physical bounds on variable $Q$ are [3]: 
$Q_{\min }=10 \mathrm{~m}^{3} / \mathrm{s}$

- $Q_{\max }=10000 \mathrm{~m}^{3} / \mathrm{s}$.

The parameters $\alpha$ and $\beta$ in (6) are affected by epistemic uncertainty; however, a large amount of data (i.e., 149 annual maximal flow values) is available for performing statistical inference on them. In particular, the point estimates $\hat{\mu}_{\alpha}$ and $\hat{\mu}_{\beta}$ and the corresponding standard deviations $\hat{\sigma}_{\alpha}$ and $\hat{\sigma}_{\beta}$ have been obtained for the parameters $\alpha$ and $\beta$ of the Gumbel distribution (6) by performing maximum likelihood estimations with the 149 data available: the method has provided $\hat{\mu}_{\alpha}=1013 \mathrm{~m}^{3} / \mathrm{s}, \hat{\mu}_{\beta}=558 \mathrm{~m}^{3} / \mathrm{s}, \hat{\sigma}_{\alpha}=48 \mathrm{~m}^{3} / \mathrm{s}$ and $\hat{\sigma}_{\beta}=36 \mathrm{~m}^{3} / \mathrm{s}$ [3]. Since a large amount of data (i.e., 149) has been used for performing statistical inference on $\alpha$ and $\beta$, then the epistemic uncertainty associated to them is mainly of "statistical nature". As a consequence, a probabilistic treatment of this epistemic uncertainty has been proposed in the original paper [3]: in particular, $\alpha$ and $\beta$ have been chosen to be normally distributed, i.e., $\alpha \sim p^{\alpha}(\alpha)=N\left(\hat{\mu}_{\alpha}, \hat{\sigma}_{\alpha}\right)=N(1013,48)$ and $\beta \sim p^{\beta}(\beta)=N\left(\hat{\mu}_{\beta}, \hat{\sigma}_{\beta}\right)=N(558,36)[3]$.

In the present paper, the Gumbel shape of the aleatory probability distributions (6) is retained but the epistemic uncertainty on the parameters is represented in possibilistic terms: this allows defining a family of probability distributions (properly bounded by plausibility and belief functions) that quantifies the expert's lack of knowledge about the parameters themselves and, thus, his/her inability to select a single probability distribution for them. To do so, the normal probability distributions $p^{\alpha}(\alpha)$ and $p^{\beta}(\beta)$ used in [3] are transformed into the possibility distributions $\pi^{\alpha}(\alpha)$ and $\pi^{\beta}(\beta)$ by normalization, i.e., $\pi^{\alpha}(\alpha)=\frac{p^{\alpha}(\alpha)}{\sup p^{\alpha}(\alpha)}, \pi^{\beta}(\beta)=\frac{p^{\beta}(\beta)}{\sup p^{\beta}(\beta)}$ [16]. The supports of the possibility distributions $\pi^{\alpha}(\alpha)$ and $\pi^{\beta}(\beta)$ are set to $\left[\hat{\mu}_{\alpha}-\hat{\sigma}_{\alpha}, \hat{\mu}_{\alpha}+\hat{\sigma}_{\alpha}\right]=[965,1061]$ and 
$\left|\hat{\mu}_{\beta}-\hat{\sigma}_{\beta}, \hat{\mu}_{\beta}+\hat{\sigma}_{\beta}\right|=[523,594]$, respectively, according to the suggestions by [3]. The possibility distributions $\pi^{\alpha}(\alpha)$ and $\pi^{\beta}(\beta)$ are shown in Figure 1, left and right, respectively.

\section{Figure 1}

Notice that in the present paper, the choice of transforming probability density functions into possibility distribution by normalization has been made arbitrarily, for the sake of simplicity, accepting that the resulting possibility distributions do not in general adhere to the probabilitypossibility consistency principle [51]; other techniques of transformation of probability density functions into possibility distributions exist, e.g., the principle of maximum specificity [52] and the principle of minimal commitment [53].

\subsubsection{The upstream and downstream riverbed levels, $Z_{m}$ and $Z_{v}$}

The minimum and maximum physical bounds on variables $Z_{m}$ and $Z_{v}$ are $Z_{m, \min }=53.5 \mathrm{~m}$, $Z_{v, \text { min }}=48 \mathrm{~m}, Z_{m, \text { max }}=57 \mathrm{~m}$ and $Z_{v, \text { max }}=51 \mathrm{~m}$, respectively [3].

Normal distributions truncated at the minimum and maximum physical bounds have been selected in [3] to represent the aleatory part of the uncertainty, i.e., $Z_{m} \sim N\left(\mu_{Z m}, \sigma_{Z m}\right)$ and $Z_{v} \sim N\left(\mu_{Z v}, \sigma_{Z v}\right)$. An amount of 29 data has been used in the reference paper [3] to provide the point estimates $\hat{\mu}_{Z m}=55.03 \mathrm{~m}, \hat{\mu}_{Z v}=50.19 \mathrm{~m}, \hat{\sigma}_{Z m}=0.45 \mathrm{~m}, \hat{\sigma}_{Z v}=0.38 \mathrm{~m}$ for parameters $\mu_{Z m}, \mu_{Z v}, \sigma_{Z m}$ and $\sigma_{Z v}$, respectively, by means of the maximum likelihood estimation method. However, according to [3] there is large uncertainty about the shape of the probability distributions of $Z_{m}$ and $Z_{v}$ : as a consequence the authors embrace a conservative "two-level" framework, using the maximum likelihood estimation method to provide also standard deviations as a measure of the uncertainty on 
the point estimates $\hat{\mu}_{Z m}, \hat{\mu}_{Z v}, \hat{\sigma}_{Z m}$ and $\hat{\sigma}_{Z v}$ : in particular, $\hat{\sigma}_{\hat{\mu}_{Z m}}=0.08, \hat{\sigma}_{\hat{\mu}_{Z v}}=0.07, \hat{\sigma}_{\hat{\sigma}_{Z m}}=0.06$ and $\hat{\sigma}_{\hat{\sigma}_{Z v}}=0.05$. Using this information, the authors in [3] model the epistemic uncertainty associated to the parameters $\mu_{Z m}, \mu_{Z v}, \sigma_{Z m}$ and $\sigma_{Z v}$ by normal distributions, i.e., $\mu_{Z m} \sim N\left(\hat{\mu}_{Z m}, \hat{\sigma}_{\hat{\mu}_{Z n}}\right), \mu_{Z v} \sim N\left(\hat{\mu}_{Z v}, \hat{\sigma}_{\hat{\mu}_{Z v}}\right), \sigma_{Z m} \sim N\left(\hat{\sigma}_{Z m}, \hat{\sigma}_{\hat{\mu}_{Z n}}\right)$ and $\sigma_{Z v} \sim N\left(\hat{\sigma}_{Z v}, \hat{\sigma}_{\hat{\sigma}_{Z v}}\right)$.

In this paper, the shapes of the aleatory probability distributions for $Z_{m}$ and $Z_{v}$, i.e., $N\left(\mu_{Z m}, \sigma_{Z m}\right)$ and $N\left(\mu_{Z v}, \sigma_{Z v}\right)$, are kept unaltered with respect to those of [3]; on the contrary, the information produced by the maximum likelihood estimation method on parameters $\mu_{Z m}, \mu_{Z v}, \sigma_{Z m}$ and $\sigma_{Z v}$, i.e., the point estimates $\hat{\mu}_{Z m}, \hat{\mu}_{Z v}, \hat{\sigma}_{Z m}, \hat{\sigma}_{Z v}$ and the corresponding standard deviations $\hat{\sigma}_{\hat{\mu}_{Z m}}, \hat{\sigma}_{\hat{\mu}_{Z v}}$, $\hat{\sigma}_{\hat{\sigma}_{Z m}}, \hat{\sigma}_{\hat{\sigma}_{Z v}}$, is used to build possibility distributions for $\mu_{Z m}, \mu_{Z v}, \sigma_{Z m}$ and $\sigma_{Z v}$ by means of the Chebyshev inequality [54], [55]. The classical Chebyshev inequality [54], [55] defines a bracketing approximation on the confidence intervals around the known mean $\mu$ of a random variable $Y$, knowing its standard deviation $\sigma$. The Chebyshev inequality can be written as follows:

$$
P(|Y-\mu| \leq k \sigma) \geq 1-\frac{1}{k^{2}} \text { for } k \geq 1
$$

Formula (7) can be thus used to define a possibility distribution $\pi$ that dominates any probability density function with given mean $\mu$ and standard deviation $\sigma$ by considering intervals $[\mu-k \sigma, \mu+k \sigma]$ as $\alpha$-cuts of $\pi$ and letting $\pi(\mu-k \sigma)=\pi(\mu+k \sigma)=\frac{1}{k^{2}}=\alpha$. This possibility distribution defines a probability family $\mathcal{P}^{\mu, \sigma}(\pi)$ which has been proven to contain all probability distributions with mean $\mu$ and standard deviation $\sigma$, whether the unknown probability distribution function is symmetric or not, unimodal or not [54].

In this case, the point estimates $\hat{\mu}_{Z m}, \hat{\mu}_{Z v}, \hat{\sigma}_{Z m}$ and $\hat{\sigma}_{Z v}$ produced by the maximum likelihood estimation method, are used in (7) as the means of the parameters $\mu_{Z m}, \mu_{Z v}, \sigma_{Z m}$ and $\sigma_{Z v}$, whereas the errors $\hat{\sigma}_{\hat{\mu}_{Z m}}, \hat{\sigma}_{\hat{\mu}_{Z v}}, \hat{\sigma}_{\hat{\sigma}_{Z m}}$ and $\hat{\sigma}_{\hat{\sigma}_{Z v}}$ associated to the estimates $\hat{\mu}_{Z m}, \hat{\mu}_{Z v}, \hat{\sigma}_{Z m}$ and $\hat{\sigma}_{Z v}$ are used in 
(7) as the standard deviations of the parameters $\mu_{Z m}, \mu_{Z v}, \sigma_{Z m}$ and $\sigma_{Z v}$ in order to build the corresponding possibility distributions $\pi^{\mu_{z n}}, \pi^{\mu_{z v}}, \pi^{\sigma_{z n}}$ and $\pi^{\sigma_{z v}}$; the supports of the possibility distributions are obtained by extending two times the standard deviation $\hat{\sigma}_{\hat{\mu}_{Z m}}, \hat{\sigma}_{\hat{\mu}_{Z v}}, \hat{\sigma}_{\hat{\sigma}_{Z m}}$ and $\hat{\sigma}_{\hat{\sigma}_{Z v}}$ in both directions with respect to the estimates $\hat{\mu}_{Z m}, \hat{\mu}_{Z v}, \hat{\sigma}_{Z m}$ and $\hat{\sigma}_{Z v}$ (Figures 2 and 3).

\section{Figure 2}

\section{Figure 3}

\subsubsection{The Strickler friction coefficient, $K_{s}$}

The Strickler friction coefficient $K_{s}$ is the most critical source of uncertainty because it is usually a simplification of a complex hydraulic model. The absolute physical limits of $K_{s}$ are $[a, b]=[5,60]$ [3].

The friction coefficient $K_{s}$ is affected by random events modifying the river status (e.g., erosion): the corresponding variability is typically described by a normal distribution, i.e., $K_{s} \sim N\left(\mu_{K s}, \sigma_{K s}\right)$ [3]. However, the mean value $\mu_{K s}$ of this normal distribution is difficult to measure because data can only be obtained through "indirect calibration characterized by significant uncertainty": in [3] this is reflected in a "very small set of five data available with $\pm 15 \%$ noise". The sample mean $\hat{\mu}_{K s}$ and standard deviation $\hat{\sigma}_{K s}$ of these five pieces of data equal 27.8 and 3, respectively. In order to reflect the imprecision generated by the indirect measurement process, the "minimal sample mean" $\hat{\mu}_{\min }=23.63$ and the "maximal sample mean" $\hat{\mu}_{\max }=31.97$ are also calculated under the conservative hypothesis that all measurements are biased in the same direction [3]. Moreover, since the small sample size adds a non-negligible "statistical epistemic uncertainty" to the values $\hat{\mu}_{\text {min }}$ 
and $\hat{\mu}_{\max }$, as described in [3] the $70 \%$ confidence bounds on $\hat{\mu}_{\min }$ and $\hat{\mu}_{\max }$ are also computed as $\hat{\mu}_{\min }-\frac{\hat{\sigma}_{K s}}{\sqrt{5}}=22.3$ and $\hat{\mu}_{\min }-\frac{\hat{\sigma}_{K s}}{\sqrt{5}}=33.3$, respectively. In [3], these considerations result in the following uncertainty quantification for $K_{s}$ :

$K_{s} \sim N\left(\mu_{K s}, \sigma_{K s}\right)$

with $\sigma_{K s}=\hat{\sigma}_{K s}=3$ and $\mu_{K s} \in\left[\hat{\mu}_{\min }-\frac{\hat{\sigma}_{K s}}{\sqrt{5}}, \hat{\mu}_{\max }+\frac{\hat{\sigma}_{K s}}{\sqrt{5}}\right]=[22.3,33.3]$.

In this paper, the shape of the aleatory probability distribution of $K_{s}$, i.e., $N\left(\mu_{K s}, \sigma_{K s}\right)$ in (8) is retained; however, differently from the original paper, a possibility distribution is associated to $\mu_{K s}$. In particular, a trapezoidal possibility distribution is here proposed: the support is chosen to be $[a, b]=\left[\hat{\mu}_{\min }-\frac{\hat{\sigma}_{K s}}{\sqrt{5}}, \hat{\mu}_{\max }+\frac{\hat{\sigma}_{K s}}{\sqrt{5}}\right]=[22.3,33.3]$ as in (8); however, in this paper additional information is provided concerning the most likely values of $\mu_{K s}$ exploiting the available data set: in particular, since the core of the trapezoidal distribution contains the most likely values of the parameter $\mu_{K s}$, in this case it is set to $[c, d]=\left[\hat{\mu}_{\min }-\frac{\hat{\sigma}_{K s}}{\sqrt{5}}, \hat{\mu}_{\max }+\frac{\hat{\sigma}_{K s}}{\sqrt{5}}\right]=[26.5,29.1]$, i.e., the interval obtained by adding/subtracting to the sample mean $\hat{\mu}_{K s}=27.8$ (which is assumed to be the most likely value for $\mu_{K s}$ ) the "statistical" epistemic uncertainty due to the low sample size (i.e., the quantity $\left.\frac{\hat{\sigma}_{K s}}{\sqrt{5}}\right)$ (Figure 4).

\section{Figure 4}

A final remark is in order with respect to the approaches considered in this work for constructing possibility distributions. The construction of the possibility distribution obviously depends on the 
information available on the uncertain parameter: when a probability distribution is originally available a corresponding possibility distribution can be generated by resorting to the probabilitypossibility transformations available in the open literature, e.g., the normalization method (like in the present case), the principle of maximum specificity or that of minimal commitment [29], [52], [53]; when the mean and the standard deviation of the parameter distribution can be estimated, e.g., by means of empirical data, the Chebyshev inequality can be used; finally, when the absolute physical limits and the most likely value(s) of the parameter are available, a triangular or trapezoidal possibility distribution can be constructed.

\section{Application}

In this Section, the hybrid method described in Section 2 is applied with the procedure in Appendix A to hierarchically propagate probabilistic and possibilistic uncertainties through the model of Section 3.1, in a "two-level" framework. The results obtained by the hybrid approach are compared to those produced by i) a traditional one-level pure probabilistic approach, where the parameters of the aleatory probability distributions are fixed, known values (only for illustration purposes, Section 4.1), ii) a MC-based DS-IRS approach, where the possibility distributions are encoded into discrete sets that are randomly and independently sampled by MC and iii) a two-level (or double loop) Monte Carlo (MC) approach, where the parameters of the aleatory probability distributions are uncertain and themselves described by probability distributions (Section 4.2).

\subsection{Comparison of the "two-level" hybrid Monte Carlo and possibilistic approach with a one-level pure probabilistic approach}

Only for illustration purposes, the following one-level pure probabilistic model has been considered for comparison: 
$Q \sim \operatorname{Gum}\left(\hat{\mu}_{\alpha}, \hat{\mu}_{\beta}\right)=\operatorname{Gum}(1013,558)$,

$Z_{m} \sim N\left(\hat{\mu}_{Z m}, \hat{\sigma}_{Z m}\right)=N(55.03,0.45)$

$Z_{v} \sim N\left(\hat{\mu}_{Z v}, \hat{\sigma}_{Z v}\right)=N(50.19,0.38)$

$K_{s} \sim N\left(\hat{\mu}_{K s}, \hat{\sigma}_{K s}\right)=N(27.8,3)$,

where the parameters of the probability distributions are defined in Sections 3.2.1 - 3.2.3: in particular, the parameters for $Q, Z_{m}$ and $Z_{v}$ correspond to their maximum likelihood estimates and the parameter $\hat{\mu}_{K s}$ of $K_{s}$ is the sample mean of the five available pieces of data obtained by neglecting measurement uncertainty.

Figure 5 shows the comparison of the cumulative distribution functions of the maximal water level of the river (i.e., the output variable of the model, $Z_{c}$ ) obtained by the one-level pure probabilistic approach (solid line) with the belief (lower dashed curve) and plausibility (upper dashed curve) functions obtained by the hybrid Monte Carlo and possibilistic approach in a "two-level" setting (Section 2 and Appendix A).

It can be seen that:

- the hybrid approach propagates the uncertainty by separating the aleatory and epistemic components; this separation is visible in the output distributions of the maximal water level of the river where the separation between the belief and plausibility functions reflects the imprecision in the knowledge of the possibilistic parameters of the probability distributions;

- the uncertainty in the output distribution of the pure probabilistic approach is given only by the slope of the cumulative distribution;

- as expected, the cumulative distribution of the maximal water level of the river obtained by the pure probabilistic method is within the belief and plausibility functions obtained by the hybrid approach.

\section{Figure 5}




\subsection{Comparison of the "two-level" hybrid Monte Carlo and possibilistic}

\section{approach with the MC-based DS-IRS and two-level (double loop) MC}

\section{approaches}

In this Section, the following approaches are considered and compared in the task of hierarchically propagating aleatory and epistemic uncertainties in a "two-level" framework:

i. $\quad$ the hybrid Monte Carlo (MC) and possibilistic approach of Section 2 and Appendix A;

ii. the Monte Carlo (MC)-based Dempster-Shafer approach employing Independent Random Sets (IRSs) (Appendix B);

iii. a two-level (double loop) MC approach (Appendix C):

a. assuming independence between the epistemically uncertain parameters of the aleatory probability distributions. This choice has been made to perform a fair comparison with the MC-based DS-IRS approach, which assumes independence between the epistemically uncertain parameters (see Appendix B);

b. assuming total dependence between the epistemically uncertain parameters of the aleatory probability distributions. This choice has been made to perform a fair comparison with the hybrid MC and possibilistic approach, which implicitly assumes by construction total dependence between the epistemically uncertain parameters (see Section 2 and Appendix A) ${ }^{2}$.

It is worth noting that the representation of epistemic uncertainty here used in the MC-based DSIRS approach entirely relies on the possibilistic representation described in Section 3.2 and

\footnotetext{
${ }^{2}$ It is important to note that the condition of total epistemic (or state-of-knowledge) dependence between parameters of risk models is far from unlikely. For example, consider the case of a system containing a number of physically distinct, but similar/ nominally identical components whose failure rates are estimated by means of the same data set: in such situation, the distributions describing the uncertainty associated to the failure rates have to be considered totally dependent [56], [57].
} 
employed by the hybrid MC and possibilistic approach: however, in order to tailor this possibilistic representation to the DS framework, the possibility distributions of Section 3.2 are discretized into focal sets (or intervals), each of which is assigned a probability mass: the reader is referred to Appendix B for some details.

In addition, notice that the probability distributions here used in the two-level MC approach for $Q$, $Z_{m}$ and $Z_{v}$ and for the corresponding epistemically uncertain parameters are the same as those proposed in the original paper by [3] (and recalled in Section 3.2.1 and 3.2.2); the only exception is represented by the probability distribution for $\mu_{K s}$, which for consistency and coherence of the comparison is here obtained by normalization of the trapezoidal possibility distribution described in Section 3.2.3 and shown in Figure 4, i.e., $p^{\mu_{K s}}\left(\mu_{K s}\right)=\frac{\pi^{\mu_{K s}}\left(\mu_{K s}\right)}{\int_{a}^{b} \pi^{\mu_{K s}}\left(\mu_{K s}\right) d \mu_{K s}}$.

Table 1 summarizes the characteristics of the approaches i. - iii. used in the following to propagate aleatory and epistemic uncertainties in a "two-level" framework.

\section{Table 1}

The following comparisons are considered: approaches that represent in the same way the epistemic uncertainty (i.e., in terms of probability or possibility distributions) but assume different relationships (i.e., dependence or independence) between the epistemically uncertain parameters are compared in Section 4.2.1 (in particular, comparisons are performed between approaches iii.a and iii.b above and between approaches i. and ii. above): such comparisons are made to study the effect of the state of dependence between the epistemically uncertain parameters of the aleatory probability distributions when a probabilistic/non-probabilistic representation of epistemic uncertainty is given; approaches assuming the same dependence relationship between the 
epistemically uncertain parameters but employing different representations of the epistemic uncertainty are compared in Section 4.2.2 (in particular, comparisons are performed between approaches ii. and iii.a above and between approaches i. and iii.b above): such comparison are made to study the effect of the probabilistic/non-probabilistic representations of the epistemically uncertain parameters of the aleatory probability distributions when the state of dependence between the epistemically uncertain parameters is given. Table 2 summarizes the comparisons carried out in the present paper together with the corresponding objectives.

\section{Table 2}

A final consideration is in order with respect to the analyses performed in the present paper. Only two extreme states of dependence between the epistemically uncertain parameters of the aleatory Probability Distribution Functions (PDFs) are here considered: in particular, independence

(methods ii. and iii.a) and total dependence (methods i. and iii.b) are assumed between all the uncertain parameters of the PDFs of all the aleatory variables. On one side, the choice of these extreme conditions serves the purpose of strongly highlighting the effects of epistemic dependence between the uncertain parameters, which allows deriving clear indications and guidelines for the application of the different approaches in risk assessment problems. On the other side, such (strong) assumptions of independence or total dependence between all the epistemically uncertain parameters may not be realistic in cases of practical interest, like the one analyzed in the present paper. Referring to the previous Section 3.2, it can be seen that the possibility distributions describing the uncertainty in the parameters of the PDFs of the four aleatory variables $Q, Z_{m}, Z_{v}$ and $K_{s}$ are estimated based on four distinct data sets (i.e., one data set for each aleatory variable). This has two implications: (1) when the PDF of a given aleatory variable contains more than one uncertain parameter (which is the case of $Q, Z_{m}$ and $Z_{v}$ ), such parameters are totally dependent 
between each other (for example, the location parameter $\alpha$ and the scale parameter $\beta$ of the PDF of variable $Q$ are totally dependent between each other because their uncertainty is estimated based on the same data set); (2) the uncertain parameters of the PDF of a given aleatory variable are epistemically independent with respect to the parameters of the PDFs of the other aleatory variables (for example, the location parameter $\alpha$ and the scale parameter $\beta$ of the PDF of variable $Q$ are independent from the mean $\mu_{Z m}$ and the standard deviation $\sigma_{Z m}$ of the PDF of variable $Z_{m}$ because their uncertainty is estimated based on two different data sets).

\subsubsection{Studying the effect of the state of dependence between the epistemically uncertain parameters of the aleatory probability distributions}

We start by comparing approaches iii.a and iii.b. above, i.e., two-level MC assuming independence and total dependence between the uncertain parameters, respectively: the upper and lower cumulative distribution functions of the model output $Z_{c}$ obtained by approaches iii.a and iii.b are shown in Figure 6.

\section{Figure 6}

In this case, assuming total dependence between the uncertain parameters is shown to lead to a smaller gap between the upper and lower cumulative distribution functions of the model output $Z_{c}$ than assuming independence. This can be easily explained by analyzing the input-output functional relationship of the model (5): it can be seen that one of the input variables (i.e., $Q$ ) appears at the numerator, whereas others (i.e., $K_{s}$ and $Z_{m}$ ) appear at the denominator, and another one appears both at the numerator and at the denominator (i.e., $Z_{v}$ ). In such a case, the highest possible values for the model output $Z_{c}$ are obtained with a combination of high values of both $Q$ and $Z_{v}$ (i.e., 
high values of the corresponding uncertain parameters $\alpha, \beta, \mu_{Z v}$ and $\sigma_{Z v}$ ) and low values of both $K_{s}$ and $Z_{m}$ (i.e., low values of the corresponding uncertain parameters $\mu_{K s}, \sigma_{K s}, \mu_{Z m}$ and $\sigma_{Z m}$ ); conversely, the lowest possible values for the model output $Z_{c}$ are obtained with a combination of low values of both $Q$ and $Z_{v}$ and high values of both $K_{s}$ and $Z_{m}$. These extreme situations (which give rise to the largest separation between the upper and lower cumulative distribution functions, i.e., to the most "epistemically" uncertain and, thus, conservative case), can be obtained only in case iii.a above, i.e., assuming independence between the epistemically uncertain parameters. Actually, if a pure random sampling is performed among independent uncertain parameters, all possible combinations of values can be in principle generated, since the entire ranges of variability of the uncertain parameters can be explored independently: thus, in some random samples, high values of $Q$ and $Z_{v}$ may be combined by chance with low values of both $K_{s}$ and $Z_{m}$, whereas in other random samples low values of both $Q$ and $Z_{v}$ may be combined by chance with high values of both $K_{s}$ and $Z_{m}$. Conversely, such "extreme" situations cannot occur if there is total dependence between the uncertain parameters (i.e., case iii.b above). Actually, in such a case high (low) values of both $Q$ and $Z_{v}$ can only be combined with high (low) values of both $K_{s}$ and $Z_{m}$, giving rise to values of output $Z_{c}$ which are lower (higher) than the highest (lowest) possible: in other words, the separation between the upper and lower cumulative distribution functions produced in case iii.b is always smaller than that produced by the "extreme" situations described above (which are possible only in case iii.a).

A final, straightforward remark is in order. The considerations made above about what combinations of parameter values would lead to the most conservative results (i.e., to the largest gap between the upper and lower cumulative distribution functions) are strictly dependent on the input-output relationship considered: obviously, a different model (with different functional relationships between inputs and outputs) would require different combinations of input values in 
order to obtain the most conservative results. For example, for the hypothetical model $w=(x * y) / z$ the most conservative results (i.e., the largest separation between the upper and lower cumulative distribution functions) would be obtained by imposing total dependence between $x$ and $y$ and opposite dependence between $z$ and both $x$ and $y$.

We now move on to compare i. and ii.. Figure 7 shows the plausibility and belief functions of the model output $Z_{c}$ produced by the MC-based DS-IRS method (case ii.) and by the hybrid MC and possibilistic approach (case i.).

\section{Figure 7}

The results are very similar because, in the present case, the effect of the different dependence relationships between the epistemically uncertain paramenters is not evident. This may be explained as follows. In general, the closer the shape of the possibility distribution of a parameter is to that of a rectangle, defined over a given support, the higher the epistemic uncertainty associated to that parameter (actually, if a parameter is represented by a rectangular possibility distribution, the only information available about the parameter is the interval where it is defined, i.e., we are totally ignorant about its distribution). It can be easily seen that if the state of knowledge of many of the epistemically uncertain parameters is close to that of total ignorance, the state of dependence between them becomes negligible. By way of example, refer to the possibility distributions of the parameters $\mu_{\mathrm{Zm}}$ (Figure 8, left) and $\beta$ (Figure 8, right) described in Section 3.2. Selecting the same confidence level $\alpha=\alpha_{1}^{\mu_{2 m}}=\alpha_{1}^{\beta}=0.5$ for the two variables (i.e., imposing total dependence between them) produces the same couple of $\alpha$-cuts than selecting different levels $\alpha_{1}^{\mu_{\mathrm{zn}}}=0.5 \neq \alpha_{2}^{\beta}=0.1$. Notice that this holds for many other combinations of $\alpha$ values: for 
example, in this case all combinations with $\alpha^{\beta}$ ranging between 0 and 0.6 and $\alpha^{\mu_{z n}}$ ranging between 0 and around 0.25 produce the same couple of $\alpha$-cuts.

\section{Figure 8}

Since, in the present case study the shape of many of the possibility distributions are quite close to that of a rectangle (see Figures 1-4), the state of dependence between the uncertain parameters scarcely affects the results.

A final consideration is in order with respect to the results obtained. The first comparison (Figure 6) shows that in the present case study the two-level MC approach assuming dependence among parameters gives rise to a smaller separation between the cumulative distribution functions than the two-level MC approach assuming independence among parameters: in other words, it can be considered less conservative. The second comparison (Figure 7) shows that the results obtained by the hybrid MC and possibilistic approach and the MC-based DS-IRS approach are very similar. Therefore, the state of dependence between the epistemically uncertain parameters of the aleatory probability distributions is more likely to become a critical factor (e.g., in risk-informed decisions) when the representation of the uncertain parameters is probabilistic.

\subsubsection{Studying the effect of the probabilistic/non-probabilistic representation of the epistemically uncertain parameters of the aleatory probability distributions}

In this Section, we perform comparisons between approaches ii. and iii.a and between approaches i. and iii.b above, i.e., approaches that represent epistemic uncertainty in radically different ways: in particular, both in hybrid and in MC-based DS-IRS methods, possibility distributions are employed 
which identify a family of probability distributions for the epistemically uncertain parameters ${ }^{3}$; on the contrary, in the two-level MC approach, only a single probability distribution is assigned to represent the epistemic uncertainty associated to the parameters.

Figure 9 shows the upper and lower cumulative distribution functions of the model output $Z_{c}$ obtained by the two-level MC approach assuming independence between the uncertain parameters (case iii.a) and the plausibility and belief functions produced by the MC-based DS-IRS approach (case ii.).

\section{Figure 9}

The results are very similar, which is explained as follows. First of all, there is obviously a strong similarity between the shapes of the probability distributions of the epistemically uncertain parameters used in the two-level MC approach (case iii.a) and the corresponding possibility distributions used in the MC-based DS-IRS approach (case ii.) ${ }^{4}$. For example, the ranges of variability of the uncertain parameters are the same for both the probability and the possibility distributions considered (see Section 3.2.1-3.2.3); in addition, some of the possibility distributions employed in the MC-based DS-IRS approach (e.g., those of parameters $\alpha$ and $\beta$ of the Gumbel distribution for $Q$ ) are obtained by simple normalization of the probability distributions employed in the two-level MC approach (Section 3.2.1); finally, the trapezoidal probability distribution used in the two-level MC approach for the Strickler friction coefficient $K_{s}$ is also obtained by simple

\footnotetext{
${ }^{3}$ Remember that in the MC-based DS-IRS approach the possibility distributions are discretized into focal sets (Appendix B).

${ }^{4}$ As before, notice that this comparison is fair because both methods assume independence between the epistemically uncertain parameters.
} 
normalization of the trapezoidal possibility distribution proposed in the present paper and shown in Figure 4 of Section 3.2.3.

In addition to the similarity between the probability and possibility distributions considered, the second motivation for the similarity between the results lies in the assumption of independence between the epistemically uncertain parameters and in the characteristics of the two algorithms used to propagate the uncertainties. In the two-level MC approach, a plain random sampling is performed from the probability distribution of the epistemically uncertain parameters, which are considered independent: as a consequence of this independence, in principle all possible combinations of values of the parameters can be sampled, since the entire ranges of variability of the parameters are explored randomly and independently. In the MC-based DS-IRS approach, the focal sets generated by the discretization of the possibility distributions are selected randomly and independently by MC (step 2. of the procedure in Appendix B); in addition, all the focal sets selected are exhaustively searched to maximize/minimize the model output.

As a final comparison, Figure 10 shows the upper and lower cumulative distribution functions of the model output $Z_{c}$ obtained by the two-level MC approach assuming total dependence between parameters (case iii.b) and the hybrid MC approach (case i.) (which assumes total dependence between parameters).

\section{Figure 10}

From the consideration made above it is clear why the gap is smaller between the cumulative distributions in the two-level MC approach assuming total dependence between the uncertain parameters (case iii.b) than between the plausibility and belief functions produced by the hybrid 
approach (case i.) $)^{5}$. Actually, in case iii.b only a limited set of combinations of uncertain parameter values can be randomly explored, whereas in case $\mathrm{i}$., the same confidence level $\alpha$ is chosen to build the $\alpha$-cuts for all the possibility distributions of the uncertain parameters (step 3. of the procedure in Appendix A). Then, the minimum and maximum values of the model output $Z_{c}$ are identified letting the uncertain parameters range independently within the corresponding $\alpha$-cuts (step 3 . of the procedure in Appendix A): thus, contrary to the case iii.b, once a possibility level $\alpha$ is selected, all possible combinations of parameter values can be explored, since the $\alpha$-cuts of all the parameters are exhaustively searched to maximize/minimize the model output $Z_{c}$ (giving rise to a larger separation between the plausibility and belief functions).

A final remark is in order with respect to the results obtained. Since in this case the hybrid MC and possibilistic approach gives rise to a larger separation between the plausibility and belief functions than the two-level MC approach (assuming total dependence between the epistemically uncertain parameters), it can be considered more conservative. As a consequence, embracing one method instead of the other may significantly change the outcome of a decision making process in a risk assessment problem involving uncertainties: this is of paramount importance in systems that are critical from the safety view point, e.g., in the nuclear, aerospace, chemical and environmental fields. On the contrary, since the results obtained by the two-level MC approach (assuming independence among the epistemically uncertain parameters) and the MC-based DS-IRS are very similar, embracing one method instead of the other would not change significantly the final decision.

In conclusion, it is worth highlighting that when there is total dependence between the epistemically uncertain parameters, a probabilistic representation of epistemic uncertainty may fail to produce

\footnotetext{
5 As before, notice that this comparison is fair because both methods assume total dependence between the epistemically uncertain parameters.
} 
reliable and conservative results, which raises concerns from the point of view of safety. A quantitative demonstration of this statement is given in what follows.

The final goal of the uncertainty propagation is to determine i) the dike level necessary to guarantee a given flood return period or ii) the flood risk for a given dike level.

With respect to issue i) above, the quantity of interest that is most relevant to the decision maker is the $99 \%$ quantile of $Z_{c}$, i.e., $Z_{c}^{0.99}$, taken as the annual maximal flood level. This corresponds to the level of a "centennial" flood, the yearly maximal water level with a 100 year-return period. With respect to issue ii) above, the quantity of interest that is most relevant to the decision maker is the probability that the maximal water level of the river $Z_{c}$ exceeds a given threshold $z^{*}$, i.e., $P\left(Z_{c} \geq z^{*}\right)$; in the present report, $z^{*}=55.5 \mathrm{~m}$ as in [3]. Table 3 reports the lower $\left(Z_{c, \text { lower }}^{0.99}\right)$ and upper $\left(Z_{c, \text { upper }}^{0.99}\right) 99^{\text {th }}$ percentiles obtained from the two limiting cumulative distributions and the corresponding LowerBound $\left(Z_{c} \geq z^{*}\right)$ and UpperBound $\left(Z_{c} \geq z^{*}\right)$. In addition, as synthetic mathematical indicators of the imprecision in the knowledge of $Z_{c}$ (i.e., of the separation between the lower and upper cumulative distribution functions), the following percentage widths have been reported:

- $W_{Z c}=\frac{Z_{c, \text { upper }}^{0.99}-Z_{c, \text { lower }}^{0.99}}{Z_{c, \text { prob }}^{0.99}}$ of the interval $\left[Z_{c, \text { lower }}^{0.99}, Z_{c, \text { upper }}^{0.99}\right]$ with respect to the percentile $Z_{c, \text { prob }}^{0.99}$ obtained by the pure probabilistic approach of Section 4.1;

- $W^{*}=\frac{\text { UpperBound }\left(Z_{c} \geq z^{*}\right)-\text { LowerBound }\left(Z_{c} \geq z^{*}\right)}{P\left(Z_{c} \geq z^{*}\right)_{\text {prob }}} \quad$ of $\quad$ the interval $\left[\right.$ LowerBound $\left(Z_{c} \geq z^{*}\right)$, UpperBound $\left.\left(Z_{c} \geq z^{*}\right)\right]$ with respect to the percentile $Z_{c, \text { prob }}^{0.99}$ obtained by the pure probabilistic approach of Section 4.1 .

Table 3 
The considerations previously reported are confirmed: there is a similarity between the values of the indicators relative to the hybrid MC and possibilistic approach (case i.), to the MC-based DS-IRS approach (case ii.) and to the two-level MC approach assuming independence among the uncertain parameters (case iii.a); on the contrary, there is a significant difference between these indicators and those produced by the two-level MC approach assuming total dependence between the uncertain parameters (case iii.b). In particular, as anticipated before, one consideration concerning the comparison between the hybrid approach and the two-level MC considering total dependence is worth to be done. Analyzing, for instance, the probability that the maximal water level of the river $Z_{c}$ exceeds the threshold $z^{*}=55.5 \mathrm{~m}, P\left[Z_{c} \geq z^{*}=55.5\right]$, it can be seen that the hybrid approach is much more conservative than the two-level MC approach assuming total dependence between parameters: in fact, for instance, the upper bounds of $P\left[Z_{c} \geq z^{*}\right]$ are 0.0241 and 0.0111 for cases $i$. and ii.b, respectively. Thus, in this case the use of the two-level MC approach would lead to underestimating by about $54 \%$ the probability that the maximal water level of the river $Z_{c}$ exceeds the threshold $z^{*}=55.5 \mathrm{~m}$ : in other words, it would lead to underestimating by about $54 \%$ the "failure probability" of the dike and, at the same time, the flood risk. The same consideration holds for the dike level necessary to guarantee a 100 year-return period represented by the $99 \%$ quantile $Z_{c}^{0.99}$ of the water level of the river; for example, the upper bounds of $Z_{c}^{0.99}$ are $56.03 \mathrm{~m}$ and $55.50 \mathrm{~m}$ for cases i. and ii.b, respectively. Thus, also in this case the use of the two-level MC approach would lead to a slight underestimation of the dike level necessary to guarantee a 100 year flood return period. Therefore, even if the two-level MC approach purposedly tries to separate variability from imprecision, differently from the hybrid approach, it treats lack-of-knowledge in the same way as it treats variability (i.e., using probability distributions): as a consequence, in some cases, it may fail to produce reliable and conservative results, which can raise great concerns from the safety point of view: in particular, in the present case study, the two-level MC approach leads to less 
conservative results when total dependence between the epistemically uncertain parameters is assumed. This leads to conclude also that when the state of dependence between the parameters is not known to the analyst (which is far from unlikely in practice), a non-probabilistic representation of epistemic uncertainty may represent the "safest" choice.

\section{Discussion of the results}

The analyses performed in the previous Section 4 can be summarized as follows:

1. a comparison between the hybrid method and the one-level pure probabilistic approach, highlighting that:

- the hybrid method explicitly propagates the uncertainty by separating the contributions coming from the aleatory and epistemic variables;

- the uncertainty in the output distribution of the pure probabilistic approach is given only by the slope of the cumulative distribution;

- as expected, the cumulative distribution of the model output obtained by the pure probabilistic method is within the belief and plausibility functions obtained by the hybrid approach;

2. comparisons between the hybrid, MC-based DS-IRS and two-level MC approaches with the following objectives:

a. the study of the effect of dependence between the epistemically uncertain parameters of the aleatory probability distributions when a probabilistic/non-probabilistic representation of epistemic uncertainty is adopted:

- the comparison between two-level MC approaches assuming total dependence and independence between the parameters, respectively, has shown that in the case study considered assuming dependence between the 
parameters leads to a smaller gap between the upper and lower cumulative distributions of the model output, i.e., to less conservative results;

- the comparison between the MC-based DS-IRS and hybrid approaches has shown that the plausibility and belief functions produced by the two approaches are similar: in other words, the hybrid method is not significantly influenced by the total dependence between the epistemically uncertain parameters, due to the large uncertainty that is associated to the parameters in the case study considered.

Based on the considerations above, it can be argued that the state of dependence between the epistemically uncertain parameters of the aleatory probability distributions is more likely to become a critical factor (e.g., in risk-informed decisions) when the representation of the uncertain parameters is probabilistic.

b. the study of the effect of the probabilistic/non-probabilistic representation of epistemic uncertainty when the state of dependence between parameters is defined:

- the comparison between the MC-based DS-IRS approach and the two-level MC approach assuming independence between the epistemically uncertain parameters has shown that in the case study considered the upper and lower cumulative distribution functions of the model output produced by the two approaches are similar. This is due to i) the strong similarity between the shapes of the possibility and probability distributions of the epistemically uncertain parameters used in the MC-based DS-IRS and two-level MC approaches, respectively, ii) the independence between the parameters and iii) the similar characteristics of the two algorithms used to propagate the uncertainties; 
- the comparison between the hybrid and the two-level MC approach assuming total dependence between the parameters has shown that the gap between the plausibility and belief functions of the model output produced by the hybrid approach is larger than the gap between the upper and lower cumulative distribution functions produced by the two-level MC method. This is due to both the different representations of epistemic uncertainties and to the characteristics of the two algorithms used to propagate the uncertainties. Actually, in the hybrid method the epistemic uncertainty on the parameters is represented by possibility distributions defining a family of probability distributions; on the contrary, in the two-level MC approach only a single probability distribution is selected to represent the epistemic uncertainty on a parameter. As a result, the two algorithms propagate the uncertainty differently: in the hybrid method, an exhaustive interval analysis is performed for different $\alpha$-cuts of the possibility distributions, whereas in the two-level MC method a plain random sampling is performed from the probability distribution of the uncertain parameters: the result is that the hybrid approach is able to explore a larger set of combinations of uncertain parameter values than the two-level MC approach (assuming dependence among parameters), thus producing more conservative results. This has been quantitatively confirmed by way of the risk model for the design of a flood protection dike through the computation of i) the dike level necessary to guarantee a 100 year flood return period and ii) the flood risk for a given dike level. In fact, both quantities have been underestimated by the two-level MC approach with respect to the hybrid approach. 
Based on the considerations above, it can be argued that a probabilistic representation of the epistemically uncertain parameters of the aleatory probability distributions may fail to produce reliable and conservative results when there is total dependence between the uncertain parameters, which raises concerns from the point of view of safety.

The findings gained by the comparisons performed in Section 4 are summarized in Table 4 for the sake of clarity.

\section{Table 4}

\section{Conclusions}

In the present paper, we performed the joint hierarchical propagation of hybrid probabilistic and possibilistic uncertainty representations onto a flood risk-based design model in a "two-level" framework. The results obtained have been compared with those produced by a one-level pure probabilistic approach, a MC-based DS-IRS approach and a two-level (double loop) MC approach with the objective of studying the effects of (i) (in)dependence between the epistemically uncertain parameters of the aleatory probability distributions and (ii) probabilistic/non-probabilistic representations of epistemic uncertainty. To the best of the authors' knowledge, this is the first time that the above mentioned methods are systematically compared with reference to risk assessment problems where hybrid uncertainty is separated into two hierarchical levels.

The findings of the work show that adopting different methods for jointly propagating hybrid uncertainties may generate different results and possibly different decisions in risk problems involving uncertainties: this is of paramount importance in systems that are critical from the safety viewpoint, e.g., in the nuclear, aerospace, chemical and environmental fields.

In particular, it seems advisable to suggest that, if nothing is known about the dependence relationship between the epistemically uncertain parameters, one should resort to the hybrid MC 
and possibilistic approach or to the MC-based DS-IRS approach because their risk estimates are more conservative than (or at least comparable to) those obtained by the two-level MC approach assuming dependence (or independence) between the epistemically uncertain parameters: thus, a non-probabilistic representation of epistemic uncertainty represents in general a "safer" choice than a probabilistic one.

\section{References}

[1] G.E. Apostolakis, The Concept of Probability in Safety Assessments of Technological Systems, Science, Vol. 250, Issue 4986, 1990, pp. 1359-1364.

[2] J.C. Helton and Oberkampf, Alternative Representations of Epistemic Uncertainty, Special Issue of Reliability Engineering and System Safety, Vol. 85, Issue 1-3, 2004, pp. 1-10.

[3] P. Limbourg, E. de Rocquigny, Uncertainty analysis using evidence theory - confronting level-1 and level-2 approaches with data availability and computational constraints, Reliability Engineering and System Safety, Vol. 95, Issue 5, pp. 550-564.

[4] A.C. Cullen, H.C. Frey, Probabilistic Techniques in Exposure Assessment: A Handbook for Dealing with Variability and Uncertainty in Models and Inputs, Plenum Press, New York, NY, 1999.

[5] K.D. Rao, H.S. Kushwaha, A.K. Verma, A. Srividya, Quantification of epistemic and aleatory uncertainties in level-1 probabilistic safety assessment studies, Reliability Engineering \& System Safety, 92(7), Pages 947-956, 2007.

[6] D.R. Karanki, H.S. Kushwaha, A.K. Verma, S. Ajit, Uncertainty Analysis Based on Probability Bounds (P-Box) Approach in Probabilistic Safety Assessment, Risk Analysis, Vol. 29, Issue 5, pp. 662-675, 2009. 
[7] T. Aven, E. Zio, Some considerations on the treatment of uncertainties in risk assessment for practical decision making, Reliability Engineering and System Safety, Vol. 96, Issue 1, pp. 64-74.

[8] G.J. Klir, B. Yuan, Fuzzy Sets and Fuzzy Logic: Theory and Applications, Prentice-Hall, Upper Saddle River, NJ, 1995.

[9] S. Ferson, V. Kreinovich, L. Ginzburg, K. Sentz, D.S. Myers, Constructing probability boxes and Dempster-Shafer structures, Sandia National Laboratories, Technical Report SAND20024015, Albuquerque, New Mexico.

[10] S. Ferson, R.B. Nelsen, J. Hajagos, D.J. Berleant, J. Zhang, W.T. Tucker, L.R. Ginzburg, W.L. Oberkampf, Dependence in probabilistic modeling, Dempster-Shafer theory, and probability bounds analysis, SAND2004.

[11] J.C. Helton, J.D. Johnson, W.L. Oberkampf, C.B. Storlie, A sampling-based computational strategy for the representation of epistemic uncertainty in model predictions with evidence theory, Computer Methods in Applied Mechanics and Engineering, 2007, 196: 3980-98.

[12] J.C. Helton, J.D. Johnson, W.L. Oberkampf, C.J. Sallaberry, Representation of Analysis Results Involving Aleatory and Epistemic Uncertainty, SAND2008-4379.

[13] K. Sentz, S. Ferson, Combination of Evidence in Dempster-Shafer Theory, Sandia National Laboratories, Technical Report SAND 2002-0835, Albuquerque, New Mexico.

[14] G. Shafer, A Mathematical Theory of Evidence, Princeton University Press, Princeton, NJ, 1976.

[15] C. Baudrit, D. Dubois, D. Guyonnet, Joint Propagation and Exploitation of Probabilistic and Possibilistic Information in Risk Assessment, IEEE Transactions on Fuzzy Systems, Vol. 14, Issue 5, 2006, pp. 593-608.

[16] C. Baudrit, D. Dubois, N. Perrot, Representing parametric probabilistic models tainted with imprecision, Fuzzy Sets and System, Vol. 159, Issue 15, 2008, pp.1913-1928. 
[17] D. Dubois, H. Prade, Possibility Theory: An Approach to Computerized Processing of Uncertainty, Plenum Press, New York, NY, 1988.

[18] D. Dubois, Possibility theory and statistical reasoning, Computational Statistics \& Data Analysis Vol 51, 2006, pp. $47-69$.

[19] S. Ferson, W.T. Tucker, Sensitivity in risk analyses with uncertain numbers, Setauket, New York 11733, SAND2006-2801

[20] S. Ferson, V. Kreinovich, J. Hajagos, W. Oberkampf and L. Ginzburg, Experimental Uncertainty Estimation and Statistics for Data Having Interval Uncertainty, Setauket, New York 11733, SAND2007-0939.

[21] R.E. Moore, Methods and Applications of Interval Analysis, SIAM, Philapdelphia, PA, 1979.

[22] M.H. Kalos, P.A. Whitlock, Monte Carlo methods. Volume I: Basics, Wiley, New York, NY, 1986.

[23] M. Marseguerra, E. Zio, Basics of the Monte Carlo Method with Application to System Reliability, Hagen, Germany: LiLoLe - Verlag GmbH, 2002.

[24] P. Baraldi, E. Zio, A Combined Monte Carlo and Possibilistic Approach to Uncertainty Propagation in Event Tree Analysis, Risk Analysis, Vol. 28, Issue 5, 2008, pp. 1309-1326.

[25] Baudrit, C., Guyonnet, D., Dubois, D., 2005. Post-processing the hybrid method for addressing uncertainty in risk assessments. Journal of the Environmental Engineering Division, ASCE 131 (12), 1750-1754.

[26] C. Baudrit, D. Guyonnet, D. Dubois, 2007. Joint propagation of variability and imprecision in assessing the risk of groundwater contamination. Journal of Contaminant Hydrology, Vol. 93, pp. 72-84.

[27] C. Baudrit, I. Couso, D. Dubois, 2007. Joint propagation of probability and possibility in risk analysis: toward a formal framework, Internat. J. Approx. Reasoning, Vol. 45, Issue 1, pp. 82105. 
[28] J.A. Cooper , S. Ferson and L. Ginzburg, 1996. Hybrid Processing of Stochastic and Subjective Uncertainty Data. Risk Analysis, Vol. 16, No. 6, pp. 785-791.

[29] R. Flage, P. Baraldi, E. Zio, T. Aven, Possibility-probability transformation in comparing different approaches to the treatment of epistemic uncertainties in a fault tree analysis, in: B. Ale, I.A. Papazoglu, E. Zio (Eds.), Reliability, Risk and Safety - Proceedings of the European Safety and Reliability (ESREL) 2010 Conference, Rhodes, Greece, 5-9 September 2010, pp. 714-721, Taylor \& Francis Group, London, United Kingdom, 2010, ISBN 978-0-415-60427-7

[30] Guyonnet, D., Bourgine, B., Dubois, D., Fargier, H., Côme, B., Chilès, J.P., 2003. Hybrid approach for addressing uncertainty in risk assessments. Journal of the Environmental Engineering Division, ASCE 129, pp. 68-78.

[31] E. Kentel, M.M. Aral, 2004. Probabilistic-fuzzy health risk modeling. Stoch. Envir. Res. and Risk Ass., Vol. 18: pp. 324-338.

[32] E. Kentel, M.M. Aral, 2007. Risk tolerance measure for decision-making in fuzzy analysis: a health risk assessment perspective. Stoch. Environ Res. Ris. Assess., Vol. 21: pp. 405-417.

[33] L.A. Zadeh, Fuzzy Sets, Information and Control, Vol. 8, Issue 3, 1965, pp. 338-353.

[34] E. Kentel, M.M. Aral, 2005. 2D Monte Carlo versus 2D Fuzzy Monte Carlo Health Risk Assessment, Internat. J. Stochastic Environ. Res. Risk Assess., 19, pp. 86-96.

[35] B. Moller, 2004. Fuzzy randomness - a contribution to imprecise probability. ZAMM - Z. Angew. Math. Mech., Vol. 84, No. 10-11, pp. 754 - 764.

[36] B. Möller, M. Beer, 2004. Fuzzy Randomness: Uncertainty in Civil Engineering and Computational Mechanics, Springer, Berlin.

[37] B. Möller, M. Beer, 2008. Engineering computation under uncertainty - Capabilities of nontraditional models. Computers and Structures, Vol. 86, pp. 1024-1041.

[38] B. Moller, W. Graf, M. Beer, 2003. Safety assessment of structures in view of fuzzy randomness. Computers and Structures, Vol. 81, pp. 1567-1582. 
[39] B. Moller, M. Beer, W. Graf, J.U. Sickert, 2006. Time-dependent reliability of textilestrengthened RC structures under consideration of fuzzy randomness. Computers and Structures, Vol. 84, pp. 585-603.

[40] C. Baudrit, D. Dubois, Comparing Methods for Joint Objective and Subjective Uncertainty Propagation with an example in a risk assessment, In : Fourth International Symposium on Imprecise Probabilities and Their Applications (ISIPTA '05), Pittsburgh, PA, USA, 20/07/2005-23/07/2005, F. G. Cozman, R. Nau, T. Seidenfeld (Eds.).

[41] C. Baudrit, D. Dubois, H. Fargier, Propagation of uncertainty involving imprecision and randomness. In: Proc. of the International Conference in Fuzzy Logic and Technology (EUSFLAT03), Zittau, Germany, 10/09/2003-12/09/2003.

[42] Fetz, T., 2001. Sets of joint probability measures generated by weighted marginal focal sets. In: Proceedings of the Second International Symposium on Imprecise Probability and Their Applications. G. de Cooman, T.L. Fine and T. Seidenfeld (eds.), Shaker Publishing, Maastricht, pp. 171-178.

[43] T. Fetz, M. Oberguggenberger, Propagation of uncertainty through multivariate functions in the framework of sets of probability measures, Reliability Engineering and System Safety, Vol. 85, pp. 73-87, 2004.

[44] J.C. Helton, J.D. Johnson, W.L. Oberkampf, An exploration of alternative approaches to the representation of uncertainty in model predictions, Reliability Engineering and System Safety, Vol. 85, pp. 39-72.

[45] S. Moral, N. Wilson, Importance sampling Monte-Carlo algorithms for the calculation of Dempster-Shafer belief, Tech. Rep. UTAI University of Granada, 1996.

[46] W.L. Oberkampf, J. C. Helton, Investigation of evidence theory for engineering applications, AIAA Non-Deterministic Approaches Forum, April 2002, Denver, Colorado, paper 20021569. 
[47] W.L. Oberkampf, J. C. Helton, K. Sentz, Mathematical Representation of Uncertainty, AIAA Non-Deterministic Approaches Forum, April 2001, Seattle, Washington, paper 2001-1645.

[48] Tonon, F., 2004. Using random set theory to propagate epistemic uncertainty through a mechanical system. Reliability Engineering and System Safety, Vol. 85, pp. 169-181.

[49] F. Tonon, A. Bernardini, A. Mammino, 2000. Determination of parameters range in rock engineering by means of Random Set Theory. Reliability Engineering and System Safety, Vol. 70, pp. $241-261$.

[50] F. Tonon, A. Bernardini, A. Mammino, 2000. Reliability analysis of rock mass response by means of Random Set Theory. Reliability Engineering and System Safety, Vol. 70, pp. 263282.

[51] Dubois D, Prade H. Fuzzy Sets and Systems: Theory and Applications. New York: Academic Press, 1980.

[52] D. Dubois, H. Prade, S. Sandri, On possibility/probability transformations. In: R. Lowen, M. Roubens (Eds.), Fuzzy Logic: State of the Art, Kluwer Academic Publishers, Dordrecht, 1993, p. $103-112$.

[53] Dubois D., Prade H., Smets P., 2008. A definition of subjective possibility. International Journal of Approximate Reasoning, 2008, Vol. 48, pp. 352-364.

[54] C. Baudrit, D. Dubois, Practical Representations of Incomplete Probabilistic Knowledge, Computational Statistics \& Data Analysis, Vol. 51, Issue 1, 2006, pp. 86-108.

[55] M. Kendall, A. Stuart, The Advanced Theory of Statistics, Griffin and Co., London, UK, 1977.

[56] G.E. Apostolakis, S. Kaplan, Pitfalls in risk calculations. Reliability Engineering, Vol. 2, Issue 2, pp. 135-145.

[57] USNRC, 2009. Guidance on the Treatment of Uncertainties Associated with PRAs in RiskInformed Decision Making. NUREG-1855, US Nuclear Regulatory Commission, Washington, DC. 


\section{Appendix A: operative procedure for the propagation of aleatory and epistemic uncertainty in the hybrid MC and possibilistic approach}

The operative steps for the propagation of hybrid probabilistic and possibilistic uncertainty in a "two-level" framework are the following:

1. sample a matrix $\left\{u_{j}^{i}\right\}, i=1,2, \cdots, m, j=1,2, \cdots, k$, of random numbers from a uniform distribution $\mathrm{U}[0,1)$;

2. set $\alpha=0$ (outer loop processing epistemic uncertainty);

3. select the $\alpha$-cuts $A_{\alpha}^{\theta_{j, 1}}, A_{\alpha}^{\theta_{j, 2}}, \ldots, A_{\alpha}^{\theta_{j, m_{j}}}$ of the possibility distributions $\boldsymbol{\pi}^{\boldsymbol{\theta}_{j}}\left(\boldsymbol{\theta}_{j}\right)=\left\{\boldsymbol{\pi}^{\theta_{j, 1}}\left(\theta_{j, 1}\right), \pi^{\theta_{j, 2}}\left(\theta_{j, 2}\right), \ldots, \pi^{\theta_{j, m_{j}}}\left(\theta_{j, m_{j}}\right)\right\}$ of the parameters $\boldsymbol{\theta}_{j}=\left\{\theta_{j, 1}, \theta_{j, 2}, \ldots, \theta_{j, m_{j}}\right\}$, of the "probabilistic" variables $Y_{1}, Y_{2}, \ldots, Y_{j}, \ldots, Y_{k}$, and the $\alpha$-cuts $A_{\alpha}^{k+1}, A_{\alpha}^{k+2}, \ldots, A_{\alpha}^{n}$ of the possibility distributions $\left\{\pi^{Y_{k+1}}\left(y_{k+1}\right), \pi^{Y_{k+2}}\left(y_{k+2}\right), \ldots, \pi^{Y_{l}}\left(y_{l}\right), \ldots, \pi^{Y_{n}}\left(y_{n}\right)\right\}$ of the "purely possibilistic" variables, $Y_{k+1}, Y_{k+2}, \ldots, Y_{l}, \ldots, Y_{n}$, as intervals of possible values $\left.\left[\underline{\boldsymbol{\theta}}_{j, \alpha}, \overline{\boldsymbol{\theta}}_{j, \alpha}\right]=\left\{\left[\underline{\theta}_{j, 1, \alpha}, \bar{\theta}_{j, 1, \alpha}\right],\left[\underline{\theta}_{j, 2, \alpha}, \bar{\theta}_{j, 2, \alpha}\right], \ldots, \underline{\theta}_{j, m_{j}, \alpha}, \bar{\theta}_{j, m_{j}, \alpha}\right\rfloor\right\}, \quad j=1,2, \cdots, k$, and $\left\lfloor\underline{y}_{l, \alpha}, \bar{y}_{l, \alpha}\right\rfloor$, $l=k+1, k+2, \cdots, n$, respectively;

4. set $i=1$ (inner loop processing aleatory uncertainty);

5. sample the $i-t h$ random intervals $\left\lfloor\underline{y}_{j, \alpha}^{i}, \bar{y}_{j, \alpha}^{i}\right\rfloor, j=1,2, \cdots, k$, of the "probabilistic" variables $Y_{j}, \quad j=1,2, \cdots, k, \quad$ corresponding to the $\alpha$-cuts $\quad\left[\underline{\boldsymbol{\theta}}_{j, \alpha}, \overline{\boldsymbol{\theta}}_{j, \alpha}\right]=$ $\left\{\left[\underline{\theta}_{j, 1, \alpha}, \bar{\theta}_{j, 1, \alpha}\right],\left[\underline{\theta}_{j, 2, \alpha}, \bar{\theta}_{j, 2, \alpha}\right], . .,\left[\underline{\theta}_{j, m_{j}, \alpha}, \bar{\theta}_{j, m_{j}, \alpha}\right]\right\}$ (found at step 3. above) and to the $i-t h$ random vector $\left\{u_{1}^{i}, u_{2}^{i}, \ldots, u_{j}^{i}, \ldots, u_{k}^{i}\right\}$ (generated at step 1 . above). In particular, the $i-t h$ 
random interval $\left\lfloor\underline{y}_{j, \alpha}^{i}, \bar{y}_{j, \alpha}^{i}\right\rfloor \quad$ for $\quad Y_{j}, j=1,2, \cdots, k, \quad$ is $\quad$ calculated by $\underline{y}_{j, \alpha}^{i}=\inf _{\boldsymbol{\theta}_{j} \in \underline{\boldsymbol{\theta}}_{j, \alpha}, \overline{\boldsymbol{\theta}}_{j, \alpha]}} F_{Y_{j}}^{-1}\left(u_{j}^{i} \mid \boldsymbol{\theta}_{j}\right)$ and $\bar{y}_{j, \alpha}^{i}=\sup _{\left.\boldsymbol{\theta}_{j} \in \underline{\boldsymbol{\theta}}_{j, \alpha} \overline{\boldsymbol{\theta}}_{j, \alpha}\right]} F_{Y_{j}}^{-1}\left(u_{j}^{i} \mid \boldsymbol{\theta}_{j}\right)$, where $F_{Y_{j}}^{-1}\left(\cdot \mid \boldsymbol{\theta}_{j}\right)$ is the inverse of the cumulative distribution function $(c d f) F_{Y_{j}}\left(\cdot \mid \boldsymbol{\theta}_{j}\right)$ of $p_{Y_{j}}\left(\cdot \mid \boldsymbol{\theta}_{j}\right)$; by way of example, Figure A. 1 shows the procedure for sampling the $i-t$ random interval $\left\lfloor\underline{y}_{j, \alpha}^{i}, \bar{y}_{j, \alpha}^{i}\right\rfloor$ for the generic uncertain variable $Y_{j}$.

6. calculate the smallest and largest values of $f\left(Y_{1}, Y_{2}, \ldots, Y_{j}, \ldots, Y_{k}, Y_{k+1}, Y_{k+2}, \ldots, Y_{l}, \ldots, Y_{n}\right)$, denoted by $\underline{f}_{\alpha}^{i}$ and $\bar{f}_{\alpha}^{i}$ respectively, letting variables $Y_{j}$ range within the intervals $\left\lfloor\underline{y}_{j, \alpha}^{i}, \bar{y}_{j, \alpha}^{i}\right\rfloor, \quad j=1,2, \cdots, k$, and letting variables $Y_{l}, \quad l=k+1, k+2, \cdots, n$ range within $\left\lfloor\underline{y}_{l, \alpha}, \bar{y}_{l, \alpha}\right\rfloor, \quad l=k+1, k+2, \cdots, n ; \quad$ in $\quad$ particular, $\underline{f}_{\alpha}^{i}=\inf _{j, Y_{j} \in\left[\underline{y}_{j, \alpha}^{i}, y_{j, \alpha}^{i}\right] l, Y_{l} \in\left[\underline{y}_{t, \alpha}, \bar{y}_{l, \alpha}\right]} f\left(Y_{1}, Y_{2}, \ldots, Y_{j}, \ldots, Y_{k}, Y_{k+1}, Y_{k+2}, \ldots, Y_{l}, \ldots, Y_{n}\right)$ and $\bar{f}_{\alpha}^{i}=\sup _{j, Y_{j} \in\left[\underline{y}_{j, \alpha}^{i}, y_{j, \alpha}^{-i}\right], l, Y_{l} \in\left[\underline{y}_{l, \alpha}, \bar{y}_{l, \alpha}\right]} f\left(Y_{1}, Y_{2}, \ldots, Y_{j}, \ldots, Y_{k}, Y_{k+1}, Y_{k+2}, \ldots, Y_{l}, \ldots, Y_{n}\right)$

7. take the values $\underline{f}_{\alpha}^{i}$ and $\bar{f}_{\alpha}^{i}$ found in 6. above as the lower and upper limits of the $\alpha$-cut of $f\left(Y_{1}, Y_{2}, \ldots, Y_{j}, \ldots, Y_{k}, Y_{k+1}, Y_{k+2}, \ldots, Y_{l}, \ldots, Y_{n}\right)$ in correspondence of the $i-t h$ random realization of the aleatory uncertainty;

8. if $i \neq m$, then set $i=i+1$ and return to step 5. above; otherwise go to step 9. below;

9. if $\alpha \neq 1$, then set $\alpha=\alpha+\Delta \alpha$ (e.g., $\Delta \alpha=0.05$ ) and return to step 3. above; otherwise, stop the algorithm: the fuzzy random realization (fuzzy interval) $\pi_{i}^{f}, i=1,2, \cdots, m$ of $Z=f\left(Y_{1}, Y_{2}, \ldots, Y_{n}\right)$ is constructed as the collection of the values $\underline{f}_{\alpha}^{i}$ and $\bar{f}_{\alpha}^{i}, i=1,2, \cdots, m$, found at step 6. above (in other words, $\pi_{i}^{f}$ is defined by all its $\alpha$-cut intervals $\left[f_{\alpha}^{i}, \bar{f}_{\alpha}^{i}\right\rfloor$ ). 
It is worth noting that performing an interval analysis on $\alpha$-cuts assumes total dependence between the epistemically uncertain variables. Actually, this procedure implies strong dependence between the information sources (e.g., the experts or observers) that supply the input possibility distributions, because the same confidence level $\alpha$ is chosen to build the $\alpha$-cuts for all the epistemically uncertain variables [15].

Finally, by way of example and only for illustration purposes, in Figure A.1 the procedure for sampling the $i-t h$ random interval $\left\lfloor\underline{y}_{j, \alpha}^{i}, \bar{y}_{j, \alpha}^{i}\right\rfloor$ for the generic uncertain variable $Y_{j}$ is shown. Let us suppose that the probability distribution of $Y_{j}$ is normal with parameters $\boldsymbol{\theta}_{j}=\left\{\theta_{j, 1}, \theta_{j, 2}\right\}=\{\mu, \sigma\}$; the mean $\mu=\theta_{j, 1}$ is represented by a triangular possibility distribution with core $c=5$ and support $[a, b]=[4,6]$ and the standard deviation $\sigma=\theta_{j, 2}$ is a fixed point-wise value $\left(\sigma=\theta_{j, 2}=4\right)$. With reference to the operative procedure outlined above, a possibility value $\alpha$ (e.g., $\alpha=0.3$ in Figure A.1, left) is selected and the corresponding $\alpha$-cut for $\mu=\theta_{j, 1}$ is found, i.e., $\left\lfloor\underline{\mu}_{\alpha}, \bar{\mu}_{\alpha}\right\rfloor=\left[\underline{\theta}_{j, 1, \alpha}, \bar{\theta}_{j, 1, \alpha}\right]=$ $[4.3,5.7]$ (see step 3 . of the procedure above). The cumulative distribution functions $F_{\boldsymbol{\theta}_{j}}^{Y_{j}}$ are constructed using the upper and lower values of $\mu$, i.e., $\underline{\mu}_{\alpha}=\underline{\theta}_{j, 1, \alpha}=4.3$ and $\bar{\mu}_{\alpha}=\bar{\theta}_{j, 1, \alpha}=5.7$

(Figure A.1, right); then, a random number $u_{j}^{i}$ (e.g., $u_{j}^{i}=0.7$ in Figure A.1, right) is sampled from a uniform distribution in $[0,1)$ and the interval $\left\lfloor\underline{y}_{j, \alpha}^{i}, \bar{y}_{j, \alpha}^{i}\right\rfloor$ is computed as $\left[\inf _{\boldsymbol{\theta}_{j} \in\left[\underline{\boldsymbol{\theta}}_{j, \alpha}, \overline{\boldsymbol{\theta}}_{j, \alpha}\right]} F_{Y_{j}}^{-1}\left(u_{j}^{i} \mid \boldsymbol{\theta}_{j}\right), \sup _{\left.\boldsymbol{\theta}_{j} \in \underline{\boldsymbol{\theta}}_{j, \alpha} \overline{\boldsymbol{\theta}}_{j, \alpha}\right]} F_{Y_{j}}^{-1}\left(u_{j}^{i} \mid \boldsymbol{\theta}_{j}\right)\right]=\left[\inf _{\mu \in\left[\underline{\mu}_{\alpha}, \bar{\mu}_{\alpha}\right]} F_{Y_{j}}^{-1}\left(u_{j}^{i} \mid \mu\right), \sup _{\mu \in\left[\underline{\mu}_{\alpha}, \bar{\mu}_{\alpha}\right]} F_{Y_{j}}^{-1}\left(u_{j}^{i} \mid \mu\right)\right]=$ $\left[\inf _{\mu \in[4.3,5.7]} F_{Y_{j}}^{-1}(0.7 \mid \mu), \sup _{\mu \in[4.3,5.7]} F_{Y_{j}}^{-1}(0.7 \mid \mu)\right]=[6.4,7.8]$ (see step 5. of the procedure above).

Figure A.1 


\section{Appendix B: operative procedure for the propagation of aleatory and epistemic uncertainty in the Monte Carlo-based Dempster-Shafer approach employing independent random sets}

In the MC-based DS-IRS approach, the possibility distributions employed in the hybrid MC and possibilistic method (Appendix A) are encoded into discrete (focal) sets as follows:

i. determine $q$ (nested) focal sets for the generic possibilistic variable/parameter $Y$ as the $\alpha$ cuts $A_{\alpha_{t}}=\left\lfloor\underline{y}_{\alpha_{t}}, \bar{y}_{\alpha_{t}}\right\rfloor, t=1,2, \ldots, q$, with $\alpha_{1}=1>\alpha_{2}>\ldots>\alpha_{q}>\alpha_{q+1}=0$;

ii. build the mass distribution of the focal sets by assigning $m_{\alpha_{t}}=\Delta \alpha_{t}=\alpha_{t}-\alpha_{t+1}$.

In particular, in the case study of the work presented in this paper, $q=20$ and $m_{\alpha_{t}}=\Delta \alpha_{t}=\Delta \alpha=0.05$, for the sake of comparison with the hybrid MC and possibilistic approach described in Section 2 and Appendix A and applied in Section 4.

The operative steps for the propagation of aleatory and epistemic uncertainty in a "two-level" framework according to the MC-based DS-IRS approach are the following ${ }^{6}$ :

1. set $i_{\alpha}=1$ (outer loop processing epistemic uncertainty);

2. sample the values $\left\{\alpha_{j, i_{p}}^{i_{\alpha}}\right\}, j=1,2, \ldots, k, i_{p}=1,2, \ldots, m_{j}$, from the discrete distribution $\left\{\left(\alpha_{j, i_{p}, t}, m_{\alpha_{j, i_{p}, t}}\right): t=1,2, \ldots, q=20\right\}=\left\{\left(\alpha_{j, i_{p}, 1}, m_{\alpha_{j, i_{p}, 1}}\right),\left(\alpha_{j, i_{p}, 2}, m_{\alpha_{j, i_{p}, 2}}\right), \ldots,\left(\alpha_{j, i_{p}, q=20}, m_{\alpha_{j, i_{p}, q=20}}\right)\right\}$ $=\{(1,0.05),(0.95,0.05), \ldots,(0,0.05)\}$; these sampled values represent the $\alpha$ levels of the focal sets of the discretized possibility distributions $\boldsymbol{\pi}^{\boldsymbol{\theta}_{j}}\left(\boldsymbol{\theta}_{j}\right)=\left\{\boldsymbol{\pi}^{\theta_{j, 1}}\left(\theta_{j, 1}\right), \pi^{\theta_{j, 2}}\left(\theta_{j, 2}\right), \ldots, \pi^{\theta_{j, m_{j}}}\left(\theta_{j, m_{j}}\right)\right\}$ of the parameters $\boldsymbol{\theta}_{j}=\left\{\theta_{j, 1}, \theta_{j, 2}, \ldots, \theta_{j, m_{j}}\right\}$ of the "probabilistic" variables $Y_{1}, Y_{2}, \ldots, Y_{j}, \ldots, Y_{k}$. Then sample the values $\left\{\alpha_{l}^{i_{\alpha}}\right\}$,

\footnotetext{
${ }^{6}$ The reader is referred to Section 2 and Appendix A for the notation used.
} 
$l=k+1, k+2, \ldots, n$,

from

the

discrete

distribution

$\left\{\left(\alpha_{l, t}, m_{\alpha_{l, t}}\right): t=1,2, \ldots, q=20\right\}=\left\{\left(\alpha_{l, 1}, m_{\alpha_{l, 1}}\right),\left(\alpha_{l, 2}, m_{\alpha_{l, 2}}\right), \ldots,\left(\alpha_{l, q=20}, m_{\alpha_{l, l=20}}\right)\right\}=\{(1,0.05)$,

$(0.95,0.05), \ldots,(0,0.05)\}$; these sampled values represent the $\alpha$ levels of the focal sets of the discretized possibility distributions $\pi^{Y_{k+1}}\left(y_{k+1}\right), \pi^{Y_{k+2}}\left(y_{k+2}\right), \ldots, \pi^{Y_{l}}\left(y_{l}\right), \ldots, \pi^{Y_{n}}\left(y_{n}\right)$ of the "purely possibilistic" variables, $Y_{k+1}, Y_{k+2}, \ldots, Y_{l}, \ldots, Y_{n}$. Notice that, differently from the hybrid MC and possibilistic approach (Appendix A), a different value $\alpha$ is randomly and independently sampled for each epistemically uncertain parameter/variable, i.e., independence is assumed between the epistemically uncertain parameters/variables;

3. on the basis of the $\alpha$ levels sampled at step 2., select the random focal sets

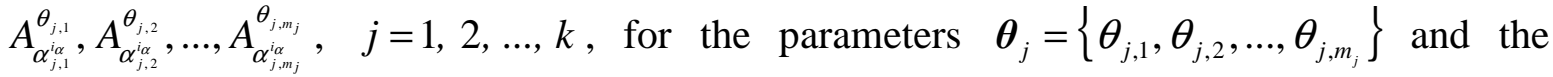
random focal sets $A_{\alpha_{k+1}^{\prime \alpha}}^{k+1}, A_{\alpha_{k+2}^{\prime \alpha}}^{k+2}, \ldots, A_{\alpha_{n}^{i \alpha}}^{n}$ for the "purely possibilistic" variables $Y_{k+1}, Y_{k+2}, \ldots, Y_{l}, \ldots, Y_{n}, \quad$ as $\quad$ intervals $\quad$ of $\quad$ values $\left.\left.\left.\left\lfloor\underline{\boldsymbol{\theta}}_{j, \alpha_{j, i_{p}}^{i \alpha}}, \overline{\boldsymbol{\theta}}_{j, \alpha_{j, i_{p}}^{\alpha_{\alpha}}}\right\rfloor=\left\{\underline{\theta}_{j, 1, \alpha_{j, 1}^{i \alpha}}, \bar{\theta}_{j, 1, \alpha_{j, 1}^{i \alpha}}\right\rfloor, \underline{\theta}_{j, 2, \alpha_{j, 2}^{i \alpha}}, \bar{\theta}_{j, 2, \alpha_{j, 2}^{i_{\alpha}}}\right\rfloor, \ldots, \underline{\theta}_{j, m_{j}, \alpha_{j, m_{j}}^{i \alpha}}, \bar{\theta}_{j, m_{j}, \alpha_{j, m_{j}}^{\alpha_{\alpha}}}\right\rfloor\right\}, \quad j=1,2, \cdots, k$, and $\left\lfloor\underline{y}_{l, \alpha_{l}^{i \alpha}}, \bar{y}_{l, \alpha_{l}^{i \alpha}}\right\rfloor, l=k+1, k+2, \cdots, n$, respectively;

4. perform the same steps 4. - 8. (inner loop processing aleatory uncertainty) as in the procedure of Appendix A to obtain $\underline{f}^{i, i_{\alpha}}$ and $\bar{f}^{i, i_{\alpha}}, i=1,2, \cdots, m, i_{\alpha}=1,2, \cdots, m_{\alpha}$, as the upper and lower limit of $f\left(Y_{1}, Y_{2}, \ldots, Y_{n}\right)$ in correspondence of the $i-t h$ random realization of the aleatory uncertainty and of the $i_{\alpha}-t h$ random realization of epistemic uncertainty;

5. if $i_{\alpha} \neq m_{\alpha}$, then set $i_{\alpha}=i_{\alpha}+1$ and return to step 2.; otherwise, stop the algorithm: the random sets $E^{i, i_{\alpha}}=\left\lfloor\underline{f}^{i, i_{\alpha}}, \bar{f}^{i, i_{\alpha}}\right\rfloor, i=1,2, \cdots, m, i_{\alpha}=1,2, \cdots, m_{\alpha}$, of $Z=f\left(Y_{1}, Y_{2}, \ldots, Y_{n}\right)$ are obtained with the collection of the values $\underline{f}^{i, i_{\alpha}}$ and $\bar{f}^{i, i_{\alpha}}, i=1,2, \cdots, m, i_{\alpha}=1,2, \cdots, m_{\alpha}$, 
found at step 5. above. A probability mass $m\left(E^{i, i_{\alpha}}\right)=\frac{1}{m_{\alpha} * m}$, is associated at each random set $E^{i, i_{\alpha}}$.

For each set $A$ contained in the universe of discourse $U_{Z}$ of the output variable $Z$, it is possible to obtain the belief $\operatorname{Bel}(A)$ and the plausibility $\operatorname{Pl}(A)$ for any set $A$, respectively [14], [15]:

$$
\begin{aligned}
& \operatorname{Bel}(A)=\sum_{E^{i, i_{\alpha}} \subseteq A} m\left(E^{i, i_{\alpha}}\right) \\
& \operatorname{Pl}(A)=\sum_{E^{i, i_{\alpha}} \cap A \neq 0} m\left(E^{i, i_{\alpha}}\right)
\end{aligned}
$$

\section{Appendix C: two-level Monte Carlo method}

Let us consider a model whose output is a function $Z=f\left(Y_{1}, Y_{2}, \ldots, Y_{j}, \ldots, Y_{n}\right)$ of $n$ uncertain variables $Y_{j}, j=1,2, \cdots, n$, that are "probabilistic", i.e., their uncertainty is described by probability distributions $\quad p_{Y_{1}}\left(y_{1} \mid \boldsymbol{\theta}_{1}\right), p_{Y_{2}}\left(y_{2} \mid \boldsymbol{\theta}_{2}\right), \ldots, p_{Y_{j}}\left(y_{j} \mid \boldsymbol{\theta}_{j}\right), \ldots, p_{Y_{k}}\left(y_{k} \mid \boldsymbol{\theta}_{k}\right) \quad$ with parameters $\boldsymbol{\theta}_{j}=\left\{\theta_{j, 1}, \theta_{j, 2}, \ldots, \theta_{j, m_{j}}\right\}, j=1,2, \cdots, n$; the parameters $\left\{\boldsymbol{\theta}_{j}: j=1,2, \cdots, n\right\}$ are themselves described by probability distributions $\boldsymbol{p}^{\boldsymbol{\theta}_{j}}\left(\boldsymbol{\theta}_{j}\right)=\left\{p^{\theta_{j, 1}}\left(\theta_{j, 1}\right), p^{\theta_{j, 2}}\left(\theta_{j, 2}\right), \ldots, p^{\theta_{j, m_{j}}}\left(\theta_{j, m_{j}}\right)\right\}$. By way of example, let $Y \sim N(\mu, \sigma)=N(\boldsymbol{\theta})=N\left(\theta_{1}, \theta_{2}\right)$ and the parameters $\boldsymbol{\theta}=\left\{\theta_{1}, \theta_{2}\right\}=\{\mu, \sigma\}$ have a normal distribution with known mean and variance, i.e., $\theta_{1}=\mu \sim N\left(\mu_{\mu}, \sigma_{\mu}\right)$ and $\theta_{2}=\sigma \sim N\left(\mu_{\sigma}, \sigma_{\sigma}\right)^{7}$.

\footnotetext{
${ }^{7}$ It is worth noting that in the following, for ease of notation, the entire set of epistemically uncertain parameters $\left\{\theta_{j, 1}, \theta_{j, 2}, \ldots, \theta_{j, m_{j}}\right\}, j=1,2, \cdots, n$, is "condensed" into a single vector $\boldsymbol{\theta}=\left\{\theta_{1}, \theta_{2}, \ldots, \theta_{k}, \ldots, \theta_{n_{p}}\right\}$, with $n_{p}=\sum_{j=1}^{n} m_{j}$, and the corresponding probability distributions are referred to as $\left\{p^{\theta_{1}}\left(\theta_{1}\right), p^{\theta_{2}}\left(\theta_{2}\right), \ldots, p^{\theta_{k}}\left(\theta_{k}\right), \ldots, p^{\theta_{n_{p}}}\left(\theta_{n_{p}}\right)\right\}$
} 
In such a case, the propagation of uncertainty can be performed by a two-level Monte Carlo (MC) technique, which is constitued by the following two main steps [2], [4]:

i. MC sampling of the parameters affected by epistemic uncertainty (outer loop processing epistemic uncertainty);

ii. repeated MC sampling of possible values of the "probabilistic" variables from the corresponding probability distributions conditioned at the values of the epistemically uncertain parameters sampled at step i. above (inner loop processing aleatory uncertainty).

In more detail, the operative steps of the procedure are:

1. set $i_{p}=1$ (outer loop processing epistemic uncertainty);

2. sample a vector $\left\{r_{k}^{i_{p}}\right\}, k=1,2, \cdots, n_{p}$ of uniform random numbers in $[0,1)\left(n_{p}\right.$ is the total number of epistemically uncertain parameters, i.e., $\left.n_{p}=\sum_{j=1}^{n} m_{j}\right)$;

3. identify the $i_{p}-t h$ set of random realizations $\theta_{k}^{i_{p}}, k=1,2, \cdots, n_{p}$, of the epistemically uncertain parameters $\theta_{k}, k=1,2, \cdots, n_{p}$, using the random vector $\left\{r_{1}^{i_{p}}, r_{2}^{i_{p}}, \ldots, r_{k}^{i_{p}}, \ldots, r_{n_{p}}^{i_{p}}\right\}$ sampled at step 2. above. In particular, the value $\theta_{k}^{i_{p}}$ is calculated by $\theta_{k}^{i_{p}}=\left[F^{\theta_{k}}\right]^{-1}\left(r_{k}^{i_{p}}\right)$ $k=1,2, \cdots, n_{p}$, where $\left[F^{\theta_{k}}\right]^{-1}$ is the inverse of the cumulative distribution $F^{\theta_{k}}$ of $p^{\theta_{k}}$;

4. set $i=1$ (inner loop processing aleatory uncertainty);

5. sample a vector $\left\{u_{j}^{i}\right\}, j=1,2, \cdots, n$, of uniform random numbers in $[0,1)$;

6. identify the $i$-th set of random realizations $y_{j}^{i, i_{p}}, j=1,2, \cdots, n$, of the "probabilistic" variables $Y_{j}, j=1,2, \cdots, n$, using the random vector $\left\{u_{1}^{i}, u_{2}^{i}, \ldots, u_{j}^{i}, \ldots, u_{n}^{i}\right\}$ sampled at step 5 . above and the random realizations $\theta_{k}^{i_{p}}, k=1,2, \cdots, n_{p}$, of the epistemically uncertain parameters sampled at step 3. above. In particular, the value $y_{j}^{i, i_{p}}$ is calculated by 
$y_{j}^{i, i_{p}}=F_{Y_{j}}^{-1}\left(u_{j}^{i} \mid \theta_{k}^{i_{p}}\right), \quad j=1,2, \cdots, n$ where $F_{Y_{j}}^{-1}\left(\cdot \mid \theta_{k}^{i_{p}}\right)$ is the inverse of the cumulative distribution $F_{Y_{j}}\left(\cdot \mid \theta_{k}^{i_{p}}\right)$ of $p_{Y_{j}}\left(\cdot \mid \theta_{k}^{i_{p}}\right)$ (notice that $p_{Y_{j}}\left(\cdot \mid \theta_{k}^{i_{p}}\right)$ is the probability distribution of $Y_{j}$ conditioned at the values $\theta_{k}^{i_{p}}, k=1,2, \cdots, n_{p}$, of the epistemically uncertain parameters $\theta_{k}, k=1,2, \cdots, n_{p}$, sampled at step 3. above;

7. calculate the value $z^{i, i_{p}}$ of the model output $Z$ as $z^{i, i_{p}}=f\left(y_{1}^{i, i_{p}}, y_{2}^{i, i_{p}}, \ldots, y_{j}^{i, i_{p}}, \ldots, y_{n}^{i, i_{p}}\right)$;

8. if $i \neq m$, then set $i=i+1$ and return to step 5.; otherwise, build the empirical cumulative distribution function $\hat{F}_{i_{p}}^{Z}$ for $Z$ using the $m$ values of $z^{i, i_{p}}=f\left(y_{1}^{i, i_{p}}, y_{2}^{i, i_{p}}, \ldots, y_{j}^{i, i_{p}}, \ldots, y_{n}^{i, i_{p}}\right)$, $i=1,2, \cdots, m$, obtained performing steps 5 . - 7.: in other words, $\hat{F}_{i_{p}}^{Z}$ is the empirical cumulative distribution function of the model output $Z$ when the epistemically uncertain parameters $\theta_{k}, k=1,2, \cdots, n_{p}$, are set to the values $\theta_{k}^{i_{p}}, k=1,2, \cdots, n_{p}$.

9. if $i_{p} \neq m_{p}$, then set $i_{p}=i_{p}+1$ and return to step 2.; otherwise, stop the algorithm: the output of the algorithm is a set of $m_{p}$ empirical cumulative distribution functions $\left\{\hat{F}_{i_{p}}^{Z}: i_{p}=1,2, \ldots, m_{p}\right\}$ for the model output $Z$. This set $\left\{\hat{F}_{i_{p}}^{Z}: i_{p}=1,2, \ldots, m_{p}\right\}$ have to be post-processed in order to obtain the upper and lower cumulative distribution functions for $Z$ : Figure C.1 shows an example of $m_{p}=10$ cumulative distribution functions (solid lines) produced by the two-level MC approach together with the corresponding upper and lower cumulative distribution functions (dashed lines).

\section{Figure C.1}


The operative steps of the two-level MC method described above assume independence between the epistemically uncertain parameters: actually, the random vector $\left\{r_{1}^{i_{p}}, r_{2}^{i_{p}}, \ldots, r_{k}^{i_{p}}, \ldots, r_{n_{p}}^{i_{p}}\right\}$ sampled at step 2. above is such that $r_{1}^{i_{p}} \neq r_{2}^{i_{p}} \neq \ldots \neq r_{k}^{i_{p}} \neq \ldots \neq r_{n_{p}}^{i_{p}}$; on the contrary, in case of total dependence, the condition $r_{1}^{i_{p}}=r_{2}^{i_{p}}=\ldots=r_{k}^{i_{p}}=\ldots=r_{n_{p}}^{i_{p}}$ have to be imposed (Figure C.2).

Figure C.2 


\section{FIGURE CAPTIONS}

Figure 1. Possibility distributions $\pi^{\alpha}(\alpha)$ (left) and $\pi^{\beta}(\beta)$ (right) of the parameters $\alpha$ and $\beta$ of the Gumbel probability distribution (6) of the maximal water flow $Q\left[\mathrm{~m}^{3} / \mathrm{s}\right]$, obtained by normalization of the probability distributions $p^{\alpha}(\alpha)$ and $p^{\beta}(\beta)$ proposed in [3]

Figure 2. Left: possibility distribution $\pi^{\mu_{Z m}}$ of $\mu_{Z m}$ constructed using Chebyshev inequality (7) with $\hat{\mu}_{Z m}=55.03$ and $\hat{\sigma}_{\hat{\mu}_{Z m}}=0.08$. Right: possibility distribution $\pi^{\sigma_{Z n}}$ of $\sigma_{Z m}$ constructed using Chebyshev inequality (7) with $\hat{\sigma}_{Z m}=0.45$ and $\hat{\sigma}_{\hat{\sigma}_{Z m}}=0.06$

Figure 3. Left: possibility distribution $\pi^{\mu_{z v}}$ of $\mu_{z v}$ constructed using Chebyshev inequality (7) with $\hat{\mu}_{z v}=50.19$ and $\hat{\sigma}_{\hat{\mu}_{Z v}}=0.07$. Right: possibility distribution $\pi^{\sigma_{Z v}}$ of $\sigma_{Z v}$ constructed using Chebyshev inequality (7) with $\hat{\sigma}_{Z v}=0.38$ and $\hat{\sigma}_{\hat{\sigma}_{Z v}}=0.05$

Figure 4. Trapezoidal possibility distribution function for the parameter $\mu_{K s}$ with support $[a, b]=[22.3,33.3]$ and core $[c, d]=[26.5,29.1]$

Figure 5. Comparison of the cumulative distribution function of the maximal water level of the river $Z_{c}$ obtained by a one-level pure probabilistic approach (solid line) with the belief (lower dashed curve) and plausibility (upper dashed curve) functions obtained by the "two-level" hybrid Monte Carlo and possibilistic approach of Section 2

Figure 6. Comparison of the upper and lower cumulative distribution functions of the maximal water level of the river $Z_{c}$ obtained by the two-level Monte Carlo approach, considering both independence and total dependence between the epistemically uncertain parameters

Figure 7. Comparison of the cumulative distribution functions of the maximal water level of the river $Z_{c}$ obtained by the Dempster-Shafer method and the hybrid method 
Figure 8. Left: possibility distribution, $\pi^{\mu_{Z m}}\left(\mu_{\mathrm{Zm}}\right)$, of the parameter $\mu_{\mathrm{Zm}}$ of the probability distribution of the variable $Z_{m}$ (Section 3.2.2); right: possibility distribution, $\pi^{\beta}(\beta)$, of the parameter $\beta$ of the probability distribution of the variable $Q$ (Section 3.2.1)

Figure 9. Comparison of the cumulative distribution functions of the maximal water level of the river $Z_{c}$ obtained by the Dempster-Shafer method and the two-level Monte Carlo method assuming independence between the epistemically uncertain parameters

Figure 10. Comparison of the cumulative distribution functions of the maximal water level of the river $Z_{c}$ obtained by the hybrid method and the two-level Monte Carlo method assuming total dependence between the epistemically uncertain parameters

Figure A.1. Left: triangular possibility distribution of the mean $\mu$ of the normal probability distribution of $Y_{j} \sim N(\mu, 4)=$ $N(\theta)$; in evidence the $\alpha$-cut of level $\alpha=0.3\left\lfloor\underline{\theta}_{j, 1, \alpha}, \bar{\theta}_{j, 1, \alpha}\right\rfloor=\left\lfloor\underline{\mu}_{\alpha}, \bar{\mu}_{\alpha}\right\rfloor=[4.3,5.7]$. Right: cumulative distribution functions of $Y_{\mathrm{j}}$ built in correspondence of the extreme values $\underline{\mu}_{\alpha}=4.3$ and $\bar{\mu}_{\alpha}=5.7$ of the $\alpha$-cut $\left.\underline{\mu}_{\alpha}, \bar{\mu}_{\alpha}\right\rfloor$ of $\mu$. The random interval $\left[\underline{y}_{j, \alpha}^{i}, \bar{y}_{j, \alpha}^{i}\right]$ (corresponding to the uniform random number $u_{j}^{i}=0.7$ ) is found using the inverse transform method Figure C.1. $m_{p}=10$ cumulative distribution functions $\hat{F}_{i_{p}}^{Z}, i_{p}=1,2, \ldots, m_{p}$, (solid lines) produced by a two-level MC approach together with the corresponding upper and lower empirical cumulative distribution functions (dashed lines)

Figure C.2. Left: random sampling of realizations of the uncertain parameters $\theta_{1}$ and $\theta_{2}$ assuming total dependence; right: random sampling of realizations of the uncertain parameters $\theta_{1}$ and $\theta_{2}$ assuming independence 
TABLES

\begin{tabular}{|c|c|c|c|}
\hline Method & $\begin{array}{l}\text { Epistemic uncertainty } \\
\text { representation }\end{array}$ & $\begin{array}{c}\text { Epistemic uncertainty } \\
\text { propagation }\end{array}$ & $\begin{array}{c}\text { State of dependence } \\
\text { between the epistemically } \\
\text { uncertain parameters }\end{array}$ \\
\hline $\begin{array}{l}\text { Hybrid MC and } \\
\text { possibilistic (i.) }\end{array}$ & Possibility distributions & Fuzzy interval analysis & Total dependence \\
\hline $\begin{array}{l}\text { MC-based DS- } \\
\text { IRS (ii.) }\end{array}$ & $\begin{array}{c}\text { Focal sets with associated } \\
\text { probability masses (discretization } \\
\text { of possibility distributions) }\end{array}$ & $\begin{array}{c}\text { Random sampling (of } \\
\text { discrete focal sets) by MC }\end{array}$ & Independence \\
\hline $\begin{array}{l}\text { Two-level MC } \\
\text { (iii.) }\end{array}$ & Probability distributions & $\begin{array}{c}\text { Random sampling (of } \\
\text { probability distributions) } \\
\text { by MC }\end{array}$ & $\begin{array}{l}\text { Independence (iii.a) / Total } \\
\text { dependence (iii.b) }\end{array}$ \\
\hline
\end{tabular}

Table 1. Characteristics of the approaches considered to propagate aleatory and epistemic uncertainties in a "two-level" framework 


\begin{tabular}{|c|c|c|c|c|c|c|}
\hline & & \multicolumn{3}{|c|}{$\begin{array}{l}\text { State of dependence between the } \\
\text { epistemically uncertain parameters }\end{array}$} & & \multirow[b]{2}{*}{ Objective } \\
\hline & & Independence & & Total dependence & & \\
\hline \multirow{5}{*}{$\begin{array}{l}\text { Representation } \\
\text { of epistemic } \\
\text { uncertainty }\end{array}$} & Probabilistic & $\begin{array}{l}\text { Two-level MC } \\
\text { (iii.a) }\end{array}$ & vs & $\begin{array}{l}\text { Two-level MC } \\
\text { (iii.b) }\end{array}$ & $\Rightarrow$ & \multirow{3}{*}{$\begin{array}{l}\text { Study the effect of the state } \\
\text { of dependence between the } \\
\text { epistemically uncertain } \\
\text { parameters of the aleatory } \\
\text { probability distributions } \\
\text { when a probabilistic/non- } \\
\text { probabilistic representation } \\
\text { of epistemic uncertainty is } \\
\text { given }\end{array}$} \\
\hline & & vs & & vs & & \\
\hline & Non-probabilistic & $\begin{array}{l}\text { MC-based DS-IRS } \\
\text { (ii.) }\end{array}$ & vs & $\begin{array}{l}\text { Hybrid MC and } \\
\text { possibilistic (i.) }\end{array}$ & $\Rightarrow$ & \\
\hline & & 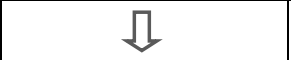 & & 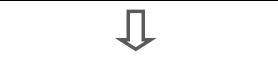 & & \\
\hline & Objective & \multicolumn{3}{|c|}{$\begin{array}{l}\text { Study the effect of the probabilistic/non- } \\
\text { probabilistic representation of the } \\
\text { epistemically uncertain parameters of the } \\
\text { aleatory probability distributions when the } \\
\text { state of dependence between the } \\
\text { epistemically uncertain parameters is given }\end{array}$} & & \\
\hline
\end{tabular}

Table 2. Comparisons performed between the different approaches, and their relative objectives 


\begin{tabular}{|c|c|c|c|c|}
\hline \multirow[t]{2}{*}{ Method } & \multicolumn{2}{|c|}{$\begin{array}{c}Z_{c}^{0.99} \\
(\text { Pure probabilistic value }= \\
55.34)\end{array}$} & \multicolumn{2}{|c|}{$\begin{array}{c}P\left[Z_{c} \geq 55.5\right] \\
\text { (Pure probabilistic value }= \\
0.0076) \\
\end{array}$} \\
\hline & $\left\lfloor Z_{c, \text { lower }}^{0.99}, Z_{c, \text { upper }}^{0.99}\right\rfloor$ & $W_{Z c}[\%]$ & $\begin{array}{l}\text { [LowerBound, } \\
\text { UpperBound] }\end{array}$ & $W *[\%]$ \\
\hline $\begin{array}{l}\text { Hybrid MC and possibilistic } \\
\text { (total dependence) (case i.) }\end{array}$ & {$[54.79,56.03]$} & 2.2 & {$[0.0024,0.0241]$} & 286 \\
\hline $\begin{array}{c}\text { MC-based DS-IRS } \\
\text { (independence) (case ii.) }\end{array}$ & {$[54.82,56.23]$} & 2.6 & {$[0.0014,0.0335]$} & 423 \\
\hline $\begin{array}{l}\text { Two-level MC (independence) } \\
\text { (case iii.a) }\end{array}$ & {$[54.56,56.06]$} & 2.7 & {$[0.0013,0.0293]$} & 368 \\
\hline $\begin{array}{c}\text { Two-level MC (total } \\
\text { dependence) (case iii.b) }\end{array}$ & {$[54.05,55.50]$} & 0.8 & {$[0.0042,0.0111]$} & 91 \\
\hline
\end{tabular}

Table 3. Comparison of the lower and upper values of $Z_{c}$ percentiles and threshold exceedance probability obtained by the three methods analyzed; the respective percentage widths $W$ of the intervals are also reported. All values are in meters 


\begin{tabular}{|c|c|c|c|c|c|c|}
\hline & & \multicolumn{3}{|c|}{$\begin{array}{l}\text { State of dependence between the } \\
\text { epistemically uncertain parameters }\end{array}$} & & \multirow[b]{2}{*}{ Findings } \\
\hline & & Independence & & $\begin{array}{c}\text { Total } \\
\text { dependence }\end{array}$ & & \\
\hline \multirow{4}{*}{ 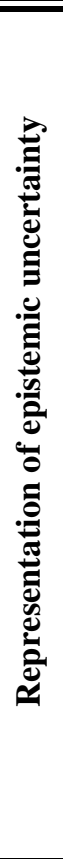 } & 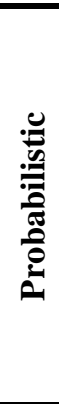 & $\begin{array}{l}\text { Two-level MC } \\
\text { (iii.a) }\end{array}$ & vs & $\begin{array}{l}\text { Two-level MC } \\
\text { (iii.b) }\end{array}$ & & \multirow{3}{*}{$\begin{array}{l}\text { Method (iii.a) vs (iii.b): } \\
\text {-In the case study considered, assuming } \\
\text { dependence between the parameters leads to a } \\
\text { smaller gap between the upper and lower CDFs } \\
\text { of the model output, i.e., to less conservative } \\
\text { results } \\
\text { Method (i) vs (ii): } \\
\text {-The plausibility and belief functions produced } \\
\text { by the two approaches are similar: in other } \\
\text { words, the hybrid method is not significantly } \\
\text { influenced by the total dependence between the } \\
\text { epistemically uncertain parameters } \\
\text { General: } \\
\text {-The state of dependence between the } \\
\text { epistemically uncertain parameters of the } \\
\text { aleatory probability distributions is more likely } \\
\text { to become a critical factor (e.g., in risk- } \\
\text { informed decisions) when the representation of } \\
\text { the uncertain parameters is probabilistic }\end{array}$} \\
\hline & & vs & & vs & & \\
\hline & 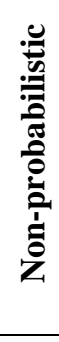 & $\begin{array}{l}\text { MC-based } \\
\text { DS-IRS (ii) }\end{array}$ & vs & $\begin{array}{l}\text { Hybrid MC and } \\
\text { possibilistic (i) }\end{array}$ & & \\
\hline & & $\downarrow$ & & $\downarrow$ & & \\
\hline & : & \multicolumn{3}{|c|}{$\begin{array}{l}\text { Method (ii) vs (iii.a): } \\
\text {-In the cases study considered, the upper and } \\
\text { lower CDFs of the model output produced by the } \\
\text { two approaches are similar } \\
\text { Method (i) vs (iii.b): } \\
\text {-The gap between the plausibility and belief } \\
\text { functions of the model output produced by the } \\
\text { hybrid approach is larger than the gap between } \\
\text { the upper and lower CDFs produced by the two- } \\
\text { level MC method } \\
\text { General: } \\
\text {-A probabilistic representation of the } \\
\text { epistemically uncertain parameters of the aleatory } \\
\text { probability distributions may fail to produce } \\
\text { reliable and conservative results when there is } \\
\text { total dependence between the uncertain } \\
\text { parameters, which raises concerns from the point } \\
\text { of view of safety }\end{array}$} & & \\
\hline
\end{tabular}

Table 4. Comparisons performed between the different approaches, and their relative findings 


\section{FIGURES}

Figure 1
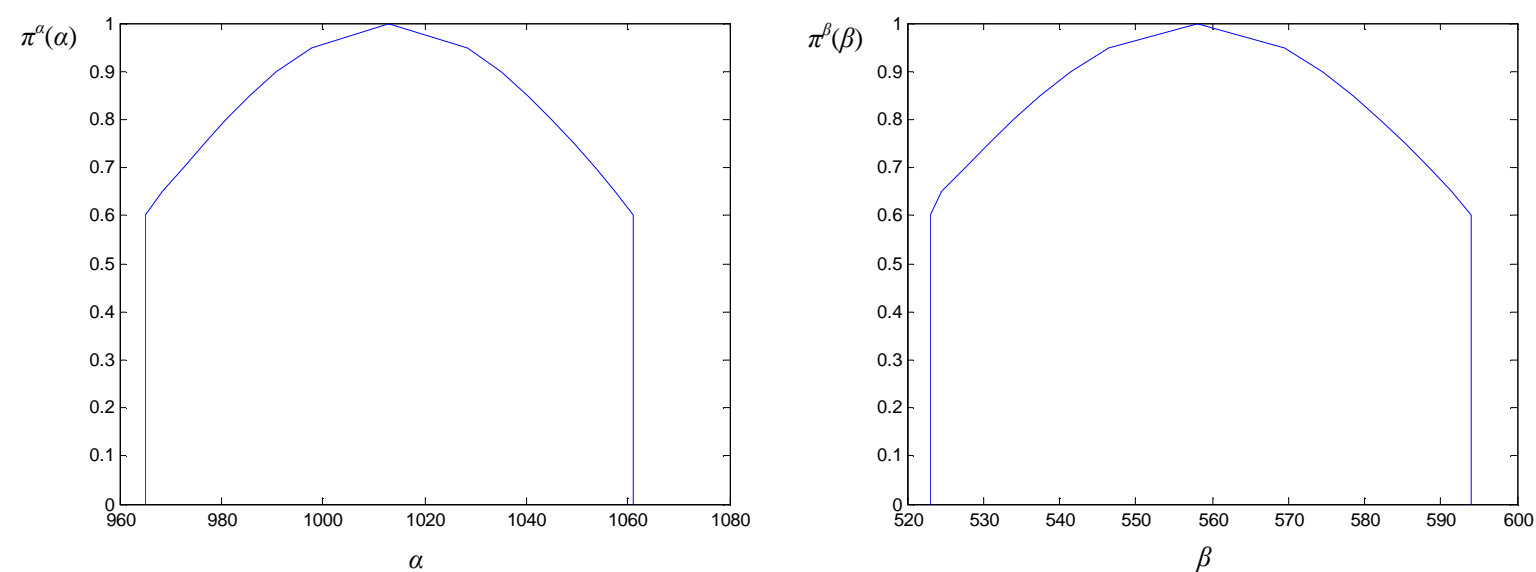
Figure 2
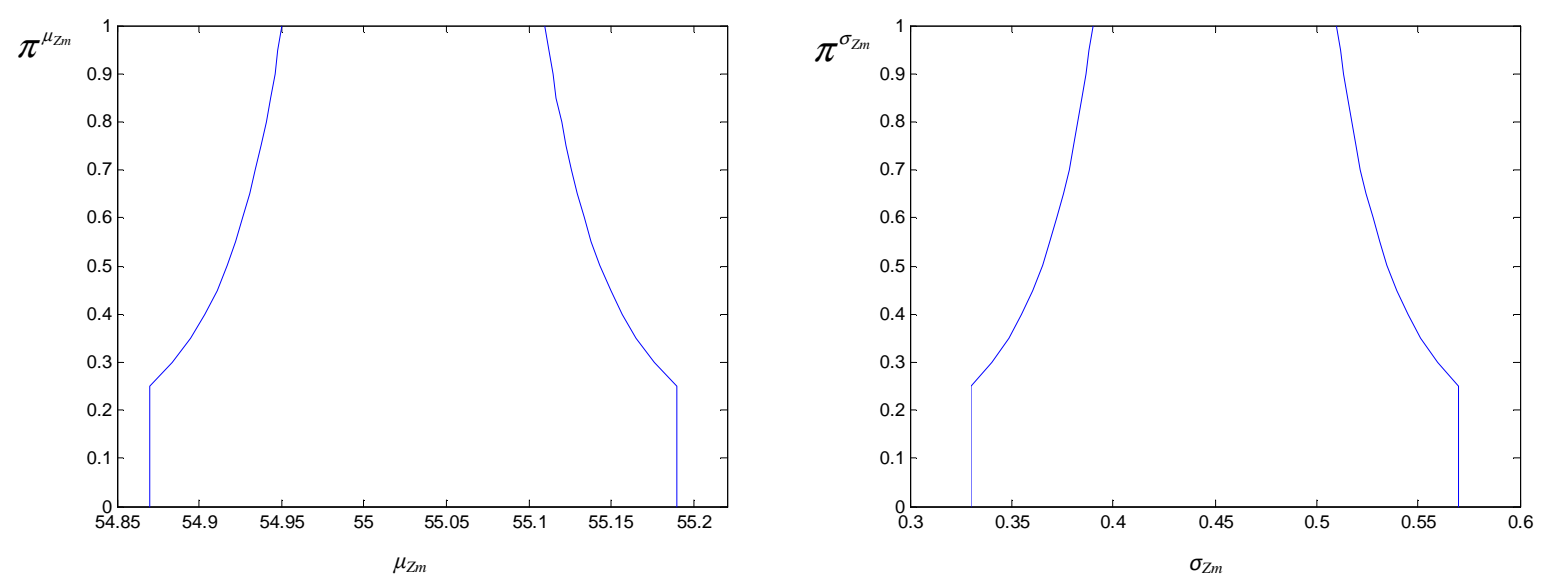
Figure 3
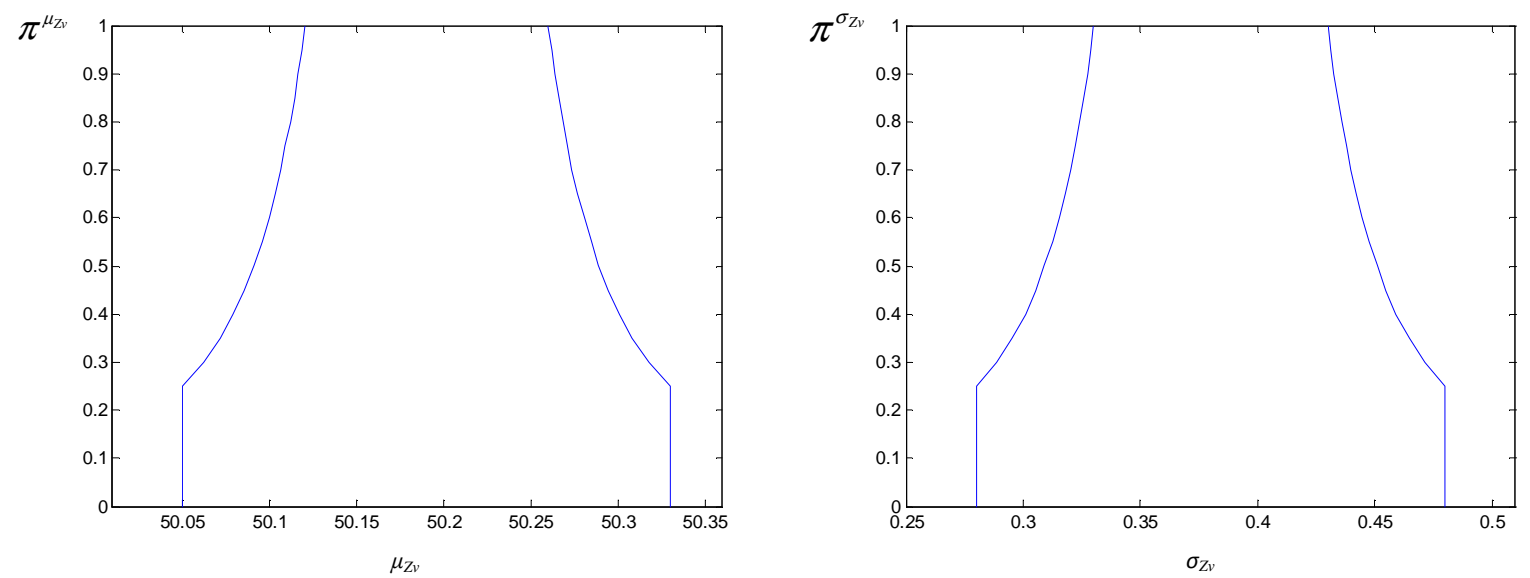
Figure 4

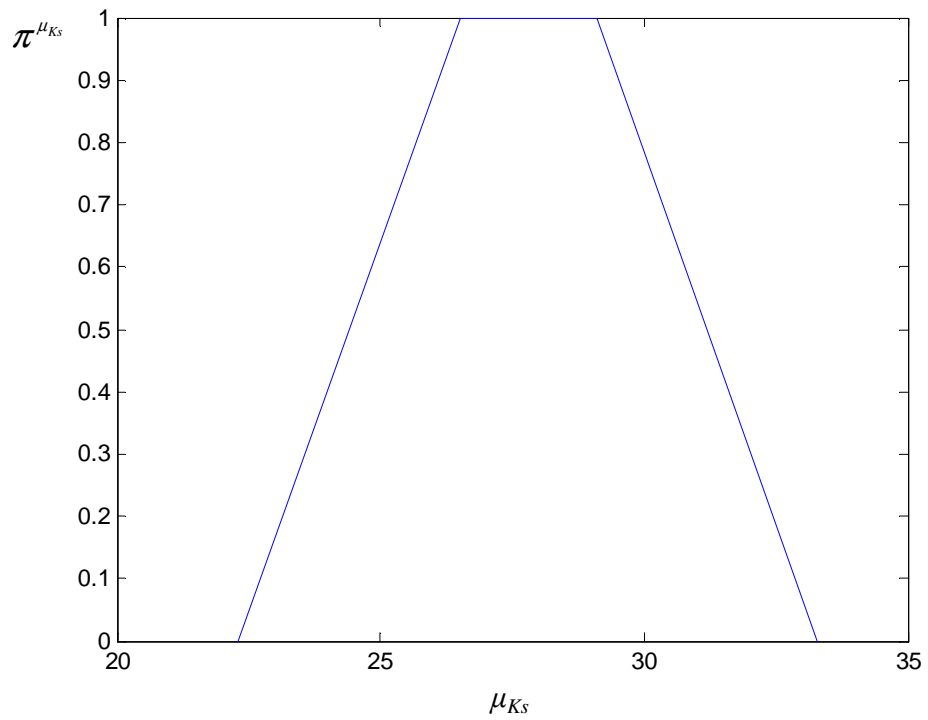


Figure 5

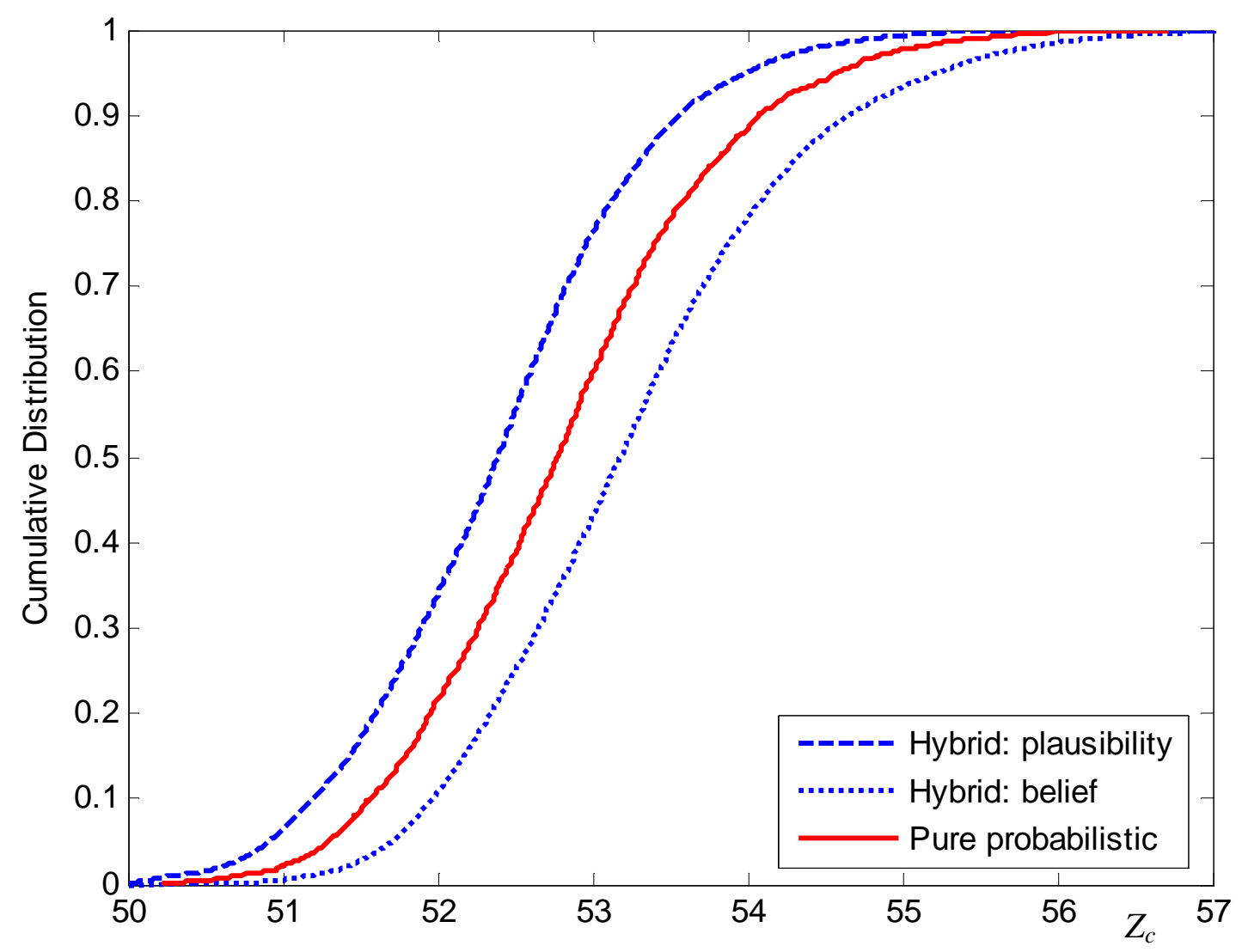


Figure 6

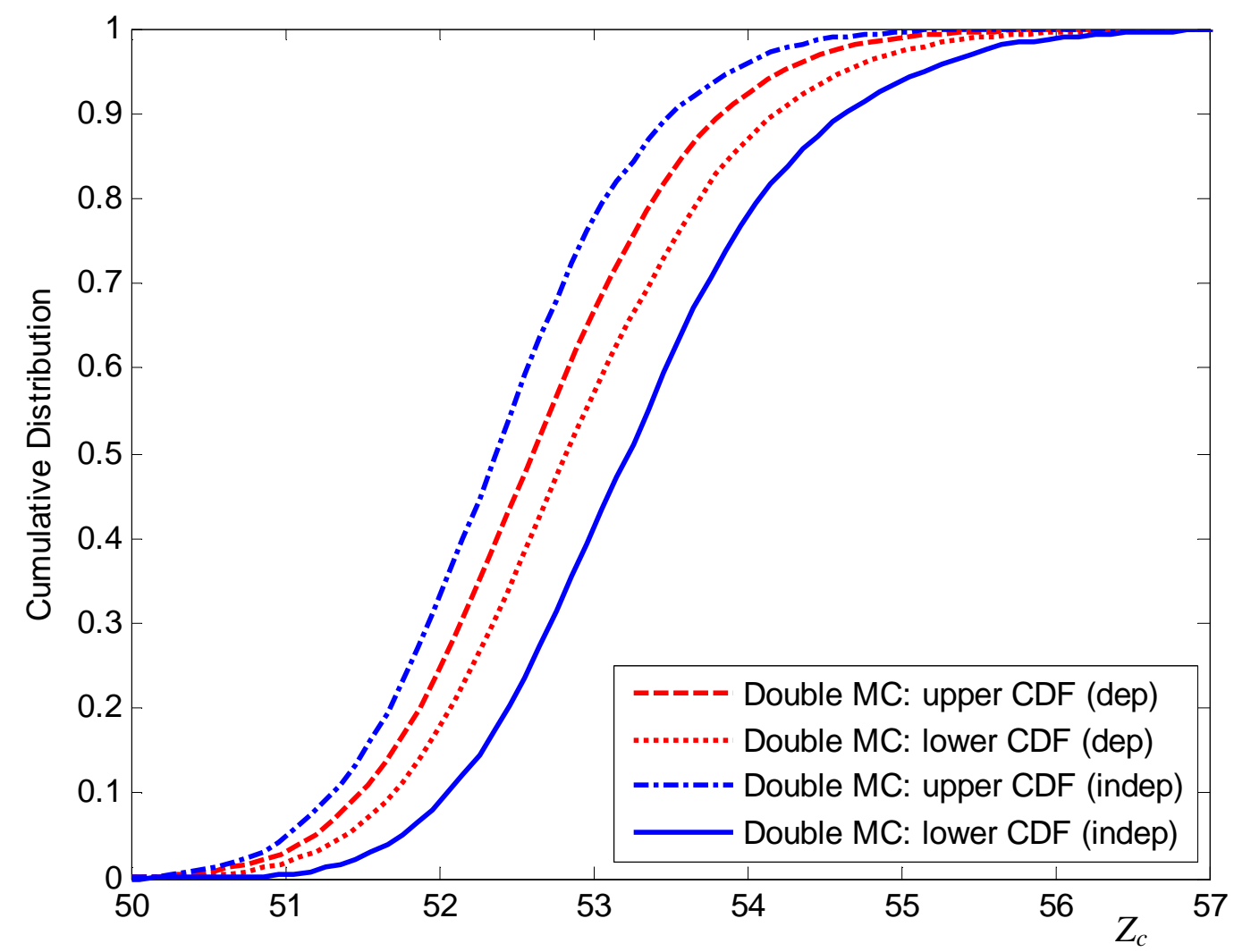


Figure 7

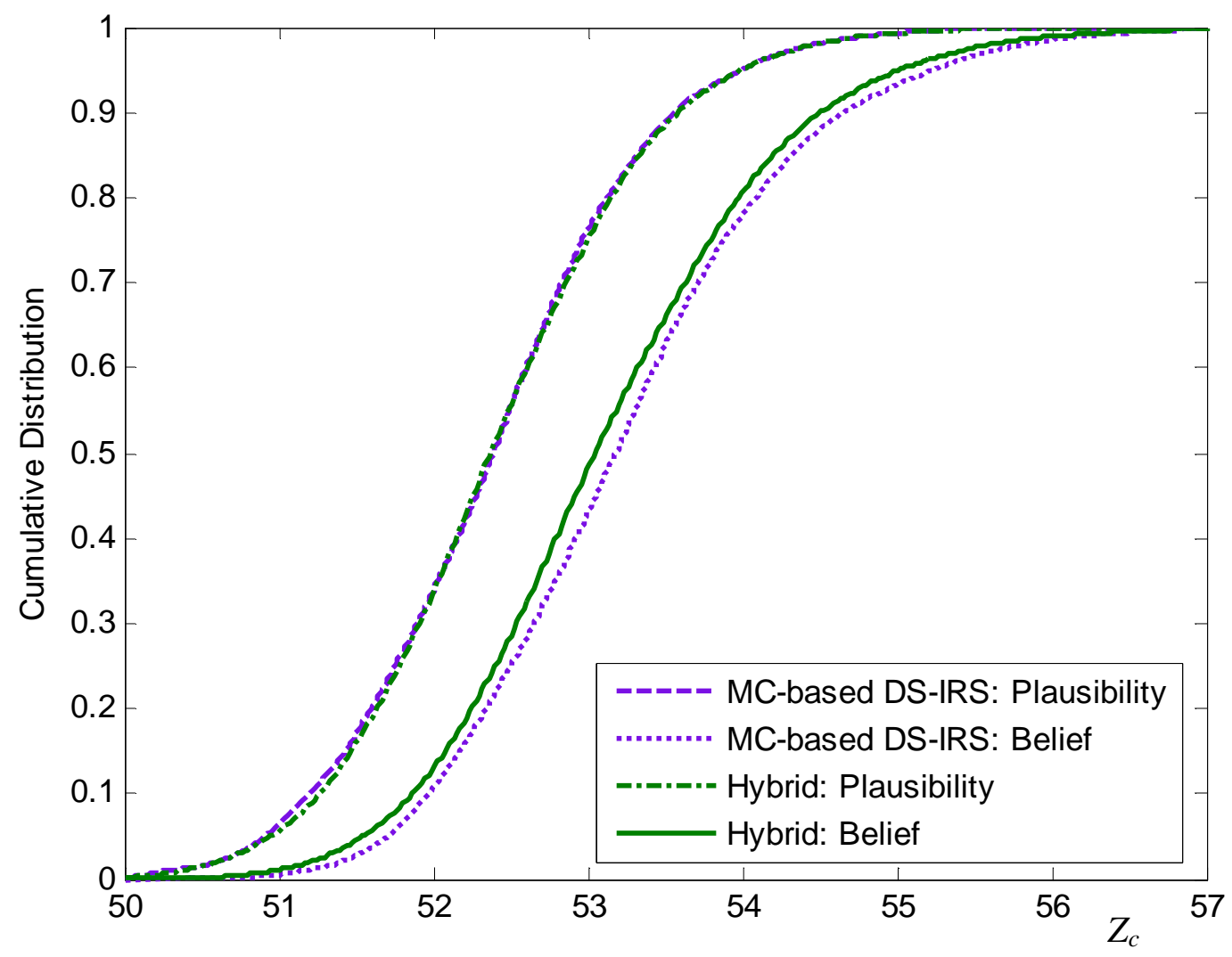




\section{Figure 8}
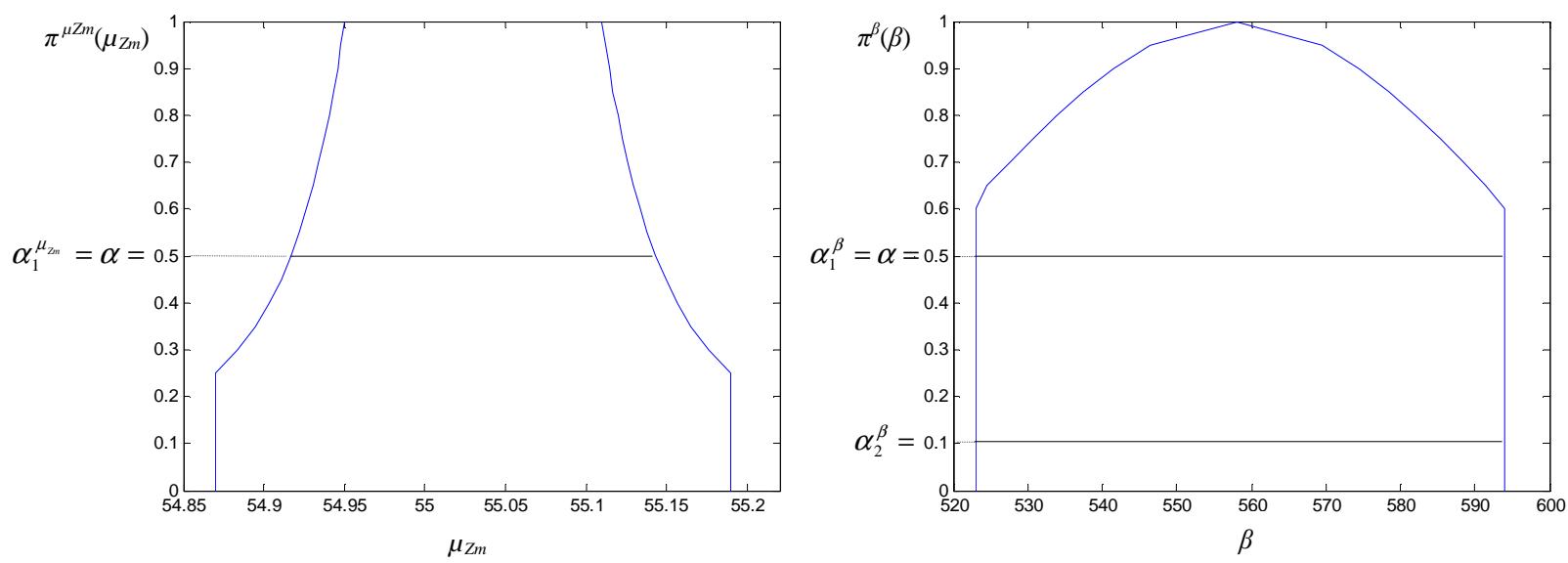
Figure 9

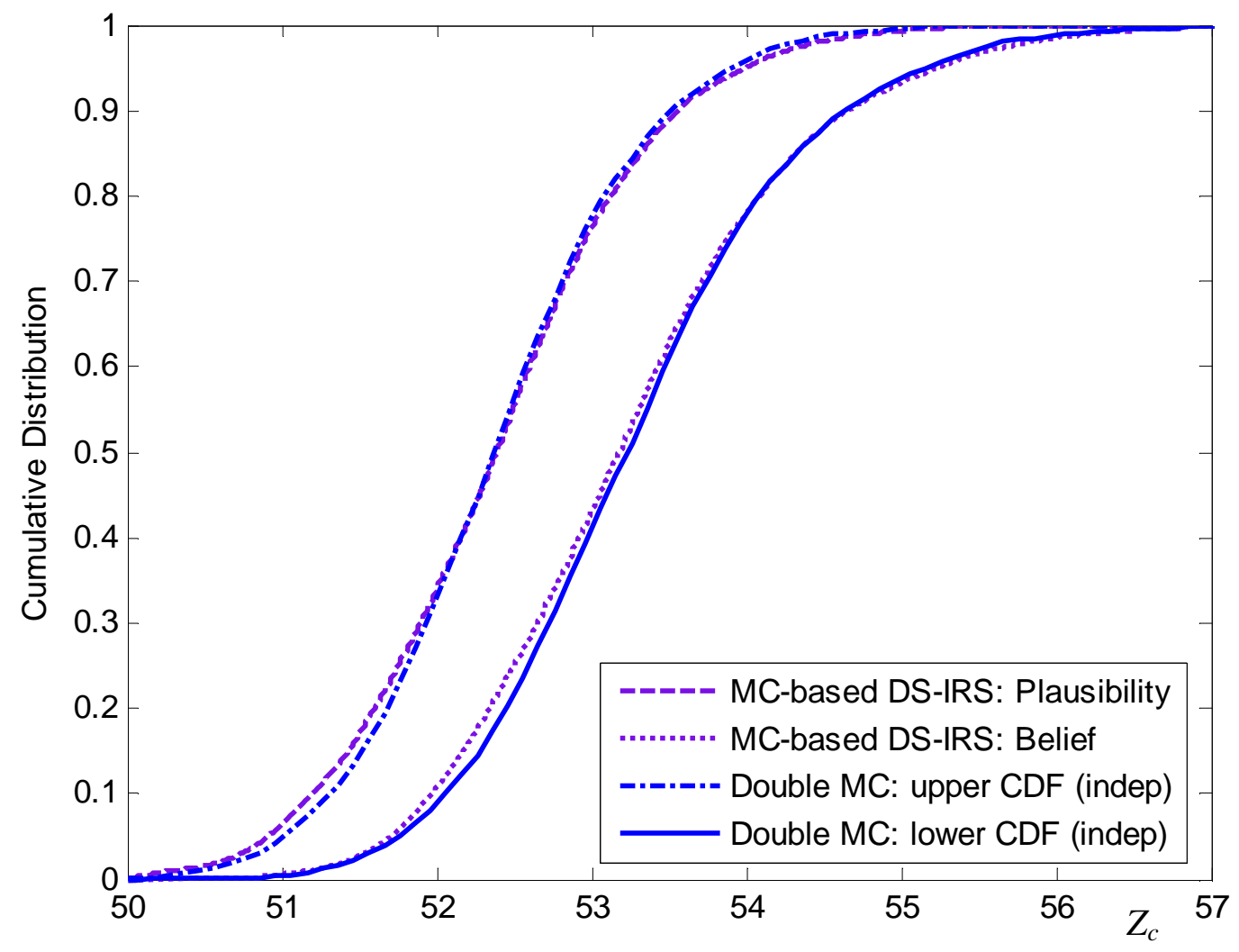


Figure 10

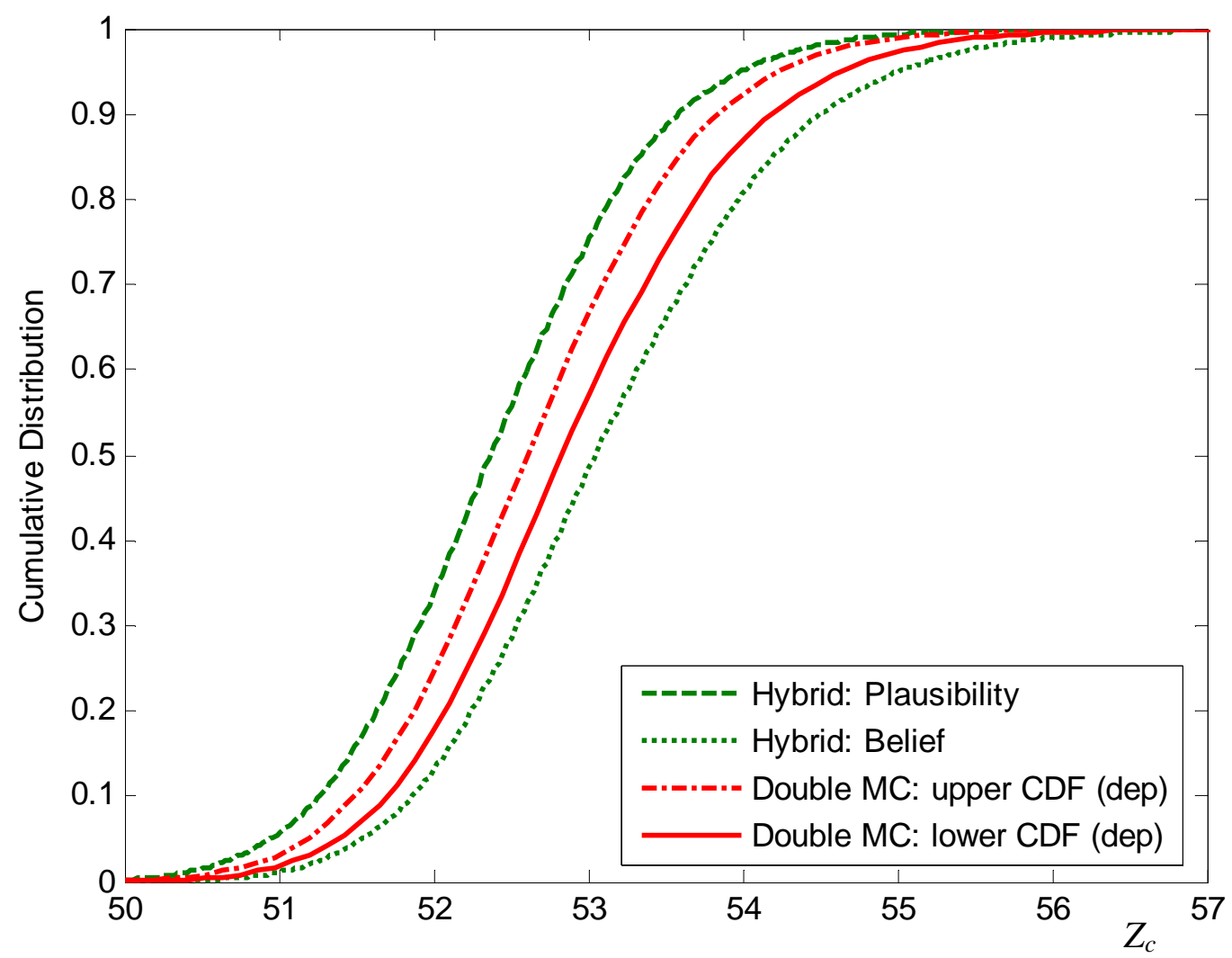


Figure A.1
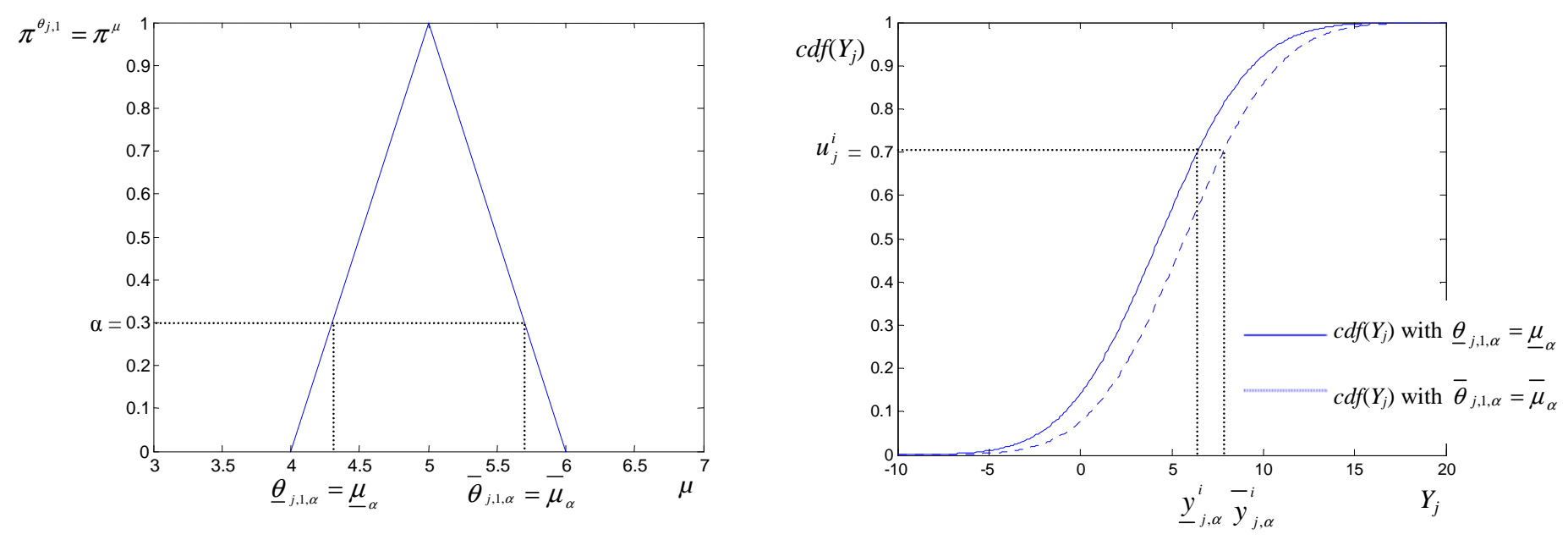
Figure C.1

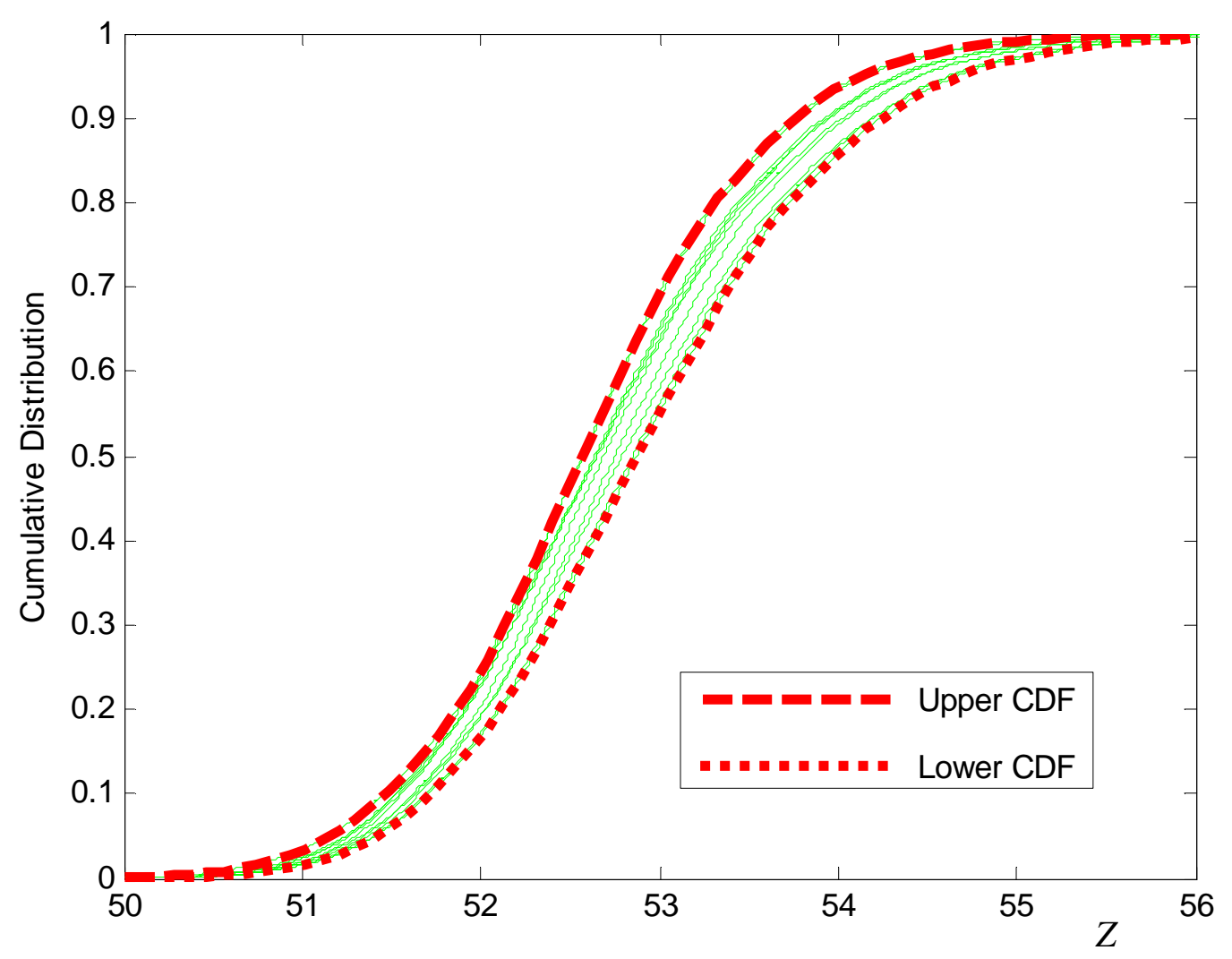


Figure C.2
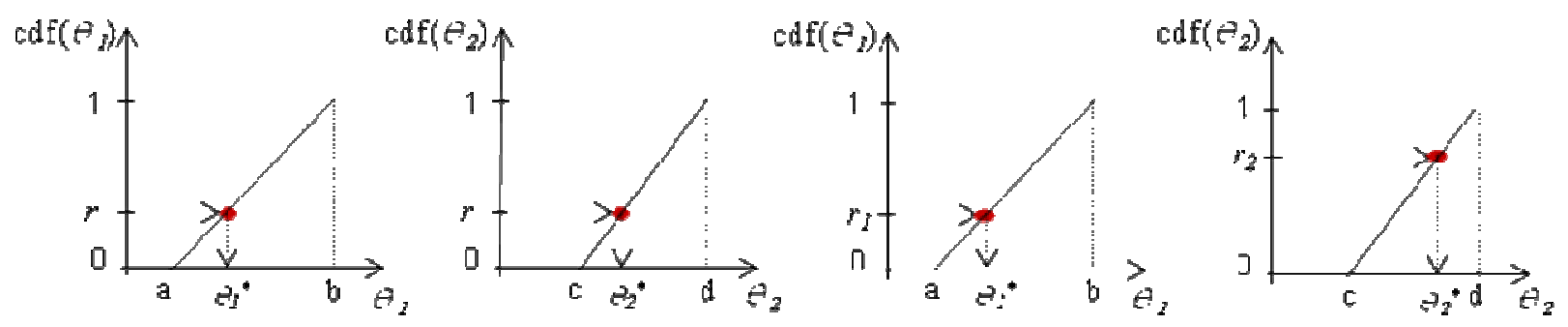